\title{
Microfluidic chips: recent advances, critical strategies in design, applications and future perspectives
}

\author{
Prapti Pattanayak ${ }^{1}$ - Sachin Kumar Singh ${ }^{1}\left[\right.$ - Monica Gulati ${ }^{1}$ - Sukriti Vishwas ${ }^{1}$ - Bhupinder Kapoor ${ }^{1}$. \\ Dinesh Kumar Chellappan ${ }^{2} \cdot$ Krishnan Anand $^{3}$. Gaurav Gupta ${ }^{4}$. Niraj Kumar Jha ${ }^{5}$. Piyush Kumar Gupta ${ }^{6}$. \\ Parteek Prasher ${ }^{7} \cdot$ Kamal Dua $^{8,9} \cdot$ Harish Dureja $^{10} \cdot$ Deepak Kumar $^{11} \cdot$ Vijay Kumar $^{12}$
}

Received: 1 July 2021 / Accepted: 19 October 2021 / Published online: 26 October 2021

(๖) The Author(s), under exclusive licence to Springer-Verlag GmbH Germany, part of Springer Nature 2021

\begin{abstract}
Microfluidic chip technology is an emerging tool in the field of biomedical application. Microfluidic chip includes a set of groves or microchannels that are engraved on different materials (glass, silicon, or polymers such as polydimethylsiloxane or PDMS, polymethylmethacrylate or PMMA). The microchannels forming the microfluidic chip are interconnected with each other for desired results. This organization of microchannels trapped into the microfluidic chip is associated with the outside by inputs and outputs penetrating through the chip, as an interface between the macro- and miniature world. With the help of a pump and a chip, microfluidic chip helps to determine the behavioral change of the microfluids. Inside the chip, there are microfluidic channels that permit the processing of the fluid, for example, blending and physicochemical responses. Microfluidic chip has numerous points of interest including lesser time and reagent utilization and alongside this, it can execute numerous activities simultaneously. The miniatured size of the chip fastens the reaction as the surface area increases. It is utilized in different biomedical applications such as food safety sensing, peptide analysis, tissue engineering, medical diagnosis, DNA purification, PCR activity, pregnancy, and glucose estimation. In the present study, the design of various microfluidic chips has been discussed along with their biomedical applications.
\end{abstract}

Keywords Microfluidic chip · Microminiaturization · Biomedical application · Microfluidic technology

Sachin Kumar Singh

singhsachin23@gmail.com; sachin_pharma06@yahoo.co.in; sachin.16030@lpu.co.in

1 School of Pharmaceutical Sciences, Lovely Professional University, Phagwara, Punjab 144411, India

2 School of Pharmacy, International Medical University, Bukit Jalil, 57000 Kuala Lumpur, Malaysia

3 Department of Chemical Pathology, School of Pathology, Faculty of Health Sciences and National Health Laboratory Service, University of the Free State, Bloemfontein, South Africa

4 School of Pharmacy, Suresh Gyan Vihar University, Mahal Road, Jagatpura, Jaipur, India

5 Department of Biotechnology, School of Engineering and Technology (SET), Sharda University, Greater Noida, Uttar Pradesh 201310, India

6 Department of Life Sciences, School of Basic Sciences and Research, Sharda University, Plot no. 32-34, Knowledge Park III, Greater Noida, Uttar Pradesh 201310, India
7 Department of Chemistry, University of Petroleum \& Energy Studies, Energy Acres, Dehradun 248007, India

8 Faculty of Health, Australian Research Centre in Complementary and Integrative Medicine, University of Technology Sydney, Ultimo, NSW 2007, Australia

9 Discipline of Pharmacy, Graduate School of Health, University of Technology Sydney, Ultimo, Australia

10 Department of Pharmaceutical Sciences, Maharshi Dayanand University, Rohtak, Haryana 12401, India

11 Department of Pharmaceutical Chemistry, School of Pharmaceutical Sciences, Shoolini University, Solan 173229, India

12 School of Bioengineering and Bioscience, Lovely Professional University, Phagwara, Punjab 144411, India 


\section{Introduction}

Microfluidic technology is a rapidly emerging field of science characterized by a range of micro-domain effects. The technology has found its place in various applicationbased streams ranging from micro-arrays to cellular biophysics. These systems essentially have a size range of $10-100 \mu \mathrm{m}$. It is also known as 'lab-on-a-chip' technology (Tabeling 2005). Microfluidic technology is accustomed to configuring miniaturized devices, which precisely controls physicochemical reactions of the fluid contained. These chips have a compact size, which in turn, increase the surface area, resulting in high mass transfer and high analytical throughput (Cui and Wang 2019; Tabeling 2005). The technology further reduces the requirement of samples and reagents, to achieve multiplexing (Cui and Wang 2019) and high-throughput screening. The grooves or microchannels that are characteristically engraved on silicon or polymer layers are specifically designed to enable efficient mixing. The common fabrication materials that are employed to prepare chips are polydimethylsiloxane (PDMS), silicon, glass, quartz, polycarbonate, and polymethylmethacrylate (PMMA) (Focaroli et al. 2014). The basic design of the microchips with their components, various types and applications are illustrated in Fig. 1. The chip contains a reagent inlet, sample inlet, valves, grooves or microchannels, the drainage system, and the sensor part. From the inlets, the reagent and sample are injected for evaluation. The flow of fluids inside the microchannels is regulated by the valves. The fluid flows through different microfluidic flows, namely open microfluidics (Berthier et al. 2016), continuous-flow (Dendukuri et al. 2006; Jahn et al. 2008), droplet-based (Seemann et al. 2011), digital-based

A.
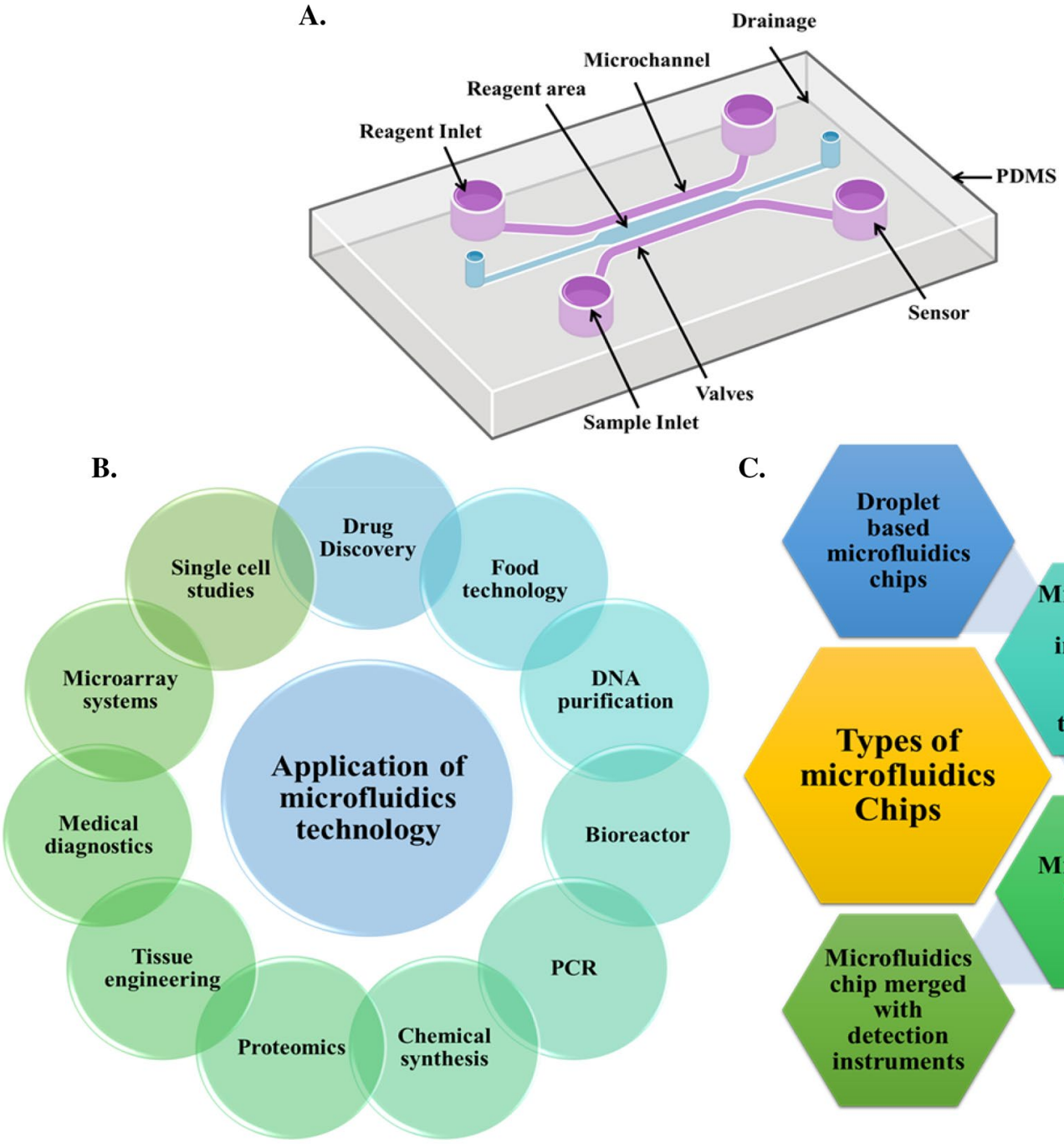

C.

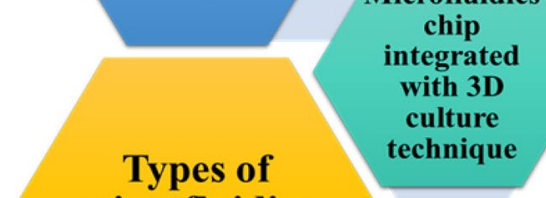

microfluidic

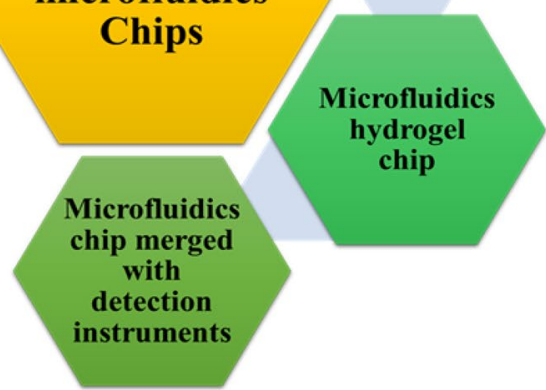

Fig. 1 A Diagrammatic representation of the microfluidic chip; $\mathbf{B}$ type of microfluidic chips and $\mathbf{C}$ application of microfluidic chips 
(Choi et al. 2012), paper-based (Lin et al. 2016), particle detection microfluidics (Xuan et al. 2010) and microfluidic-assisted magnetophoresis (Yan et al. 2016). From the drainage system, the waste material is emptied out and the sensor assists with assessing the outcomes.

\section{Types of microfluidic chips based on device configuration}

There are different types of microfluidic chips are designed, namely droplet microfluidic chip (Mashaghi et al. 2016; Seemann et al. 2011), organ-on-chip (OOC) (Wu et al. 2020), microfluidic hydrogel chip (Cuchiara et al. 2010; Nie et al. 2018), microfluidic chip integrated with 3D culture technology (Carvalho et al. 2015; Van Duinen et al. 2015; Xu et al. 2013), microfluidic chip merged with detection instruments (Gao et al. 2013; Killeen et al. 2003; Vollmer et al. 2006), microfluidic chip for single-cell analysis (Wheeler et al. 2003; Yin and Marshall 2012; Yue and Xue-Feng 2006) and microfluidic model organism (Rohde et al. 2007). These are schematically illustrated in Fig. 1B.

\subsection{Droplet-based microfluidics (DBM) chips}

A droplet-based microfluidics (DBM) system is primarily accustomed to exploit the distinct volume of fluids that are immiscible in nature (Seemann et al. 2011; Teh et al. 2008). It is among the most successful domains of microfluidic technology that deals with the manipulation of uniformly arranged microdroplets, especially at higher rates. The distinctive quality of this technique is its high production rate (Teh et al. 2008). It typically enables the formation of thousand droplets each second. In comparison with conventional techniques, it helps in producing larger number of assays and also gives a higher analysis (Zhao et al. 2017). With this technique, the sizes of microdroplets are adjustable according to their requirements. Trapping of individual cells within the droplets enables high throughput (Mashaghi et al. 2016; Teh et al. 2008).

DBM may further remove the probability of cross-contamination. The miniaturized droplets are actually microreactors that could trap anything that we intend to observe in, namely cosmetics (Opalski et al. 2019; Tan and Lee 2005), diagnostic tests and assays (Kaler and Prakash 2014; Kang et al. 2014; Srinivasan et al. 2003), low-fat food with air-filled fat particles (Chung et al. 2013; Nguyen et al. 2019), and controlled delivery of drugs with biodegradable microparticles (Liu et al. 2017a; b; Xu et al. 2009). Figure $2 \mathrm{~A}$ shows a basic design of a droplet-based microfluidics system.

\subsubsection{Formation of droplets}

Droplet formation constitutes the significant part in DBM to achieve any desired analysis. For this, two phases are necessary, one being the continuous phase and the other being the dispersed phase. There are two different types of methods

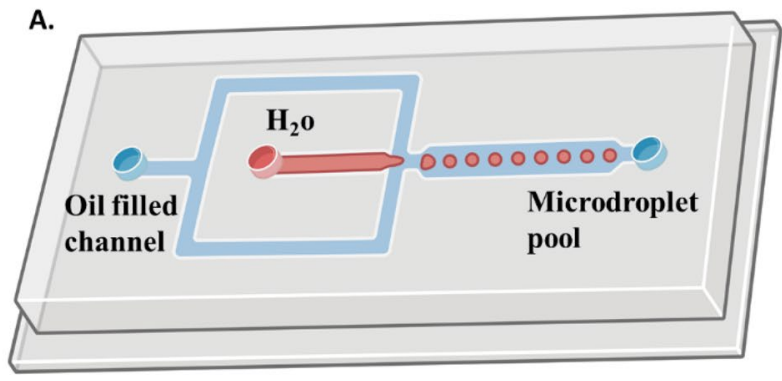

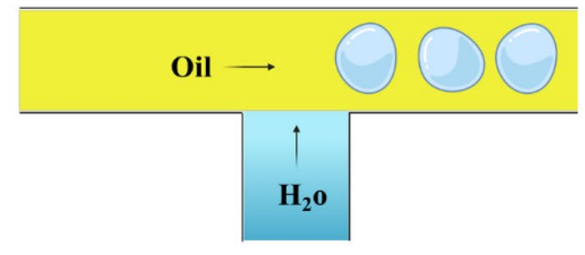

Cross-Flowing/T Junction

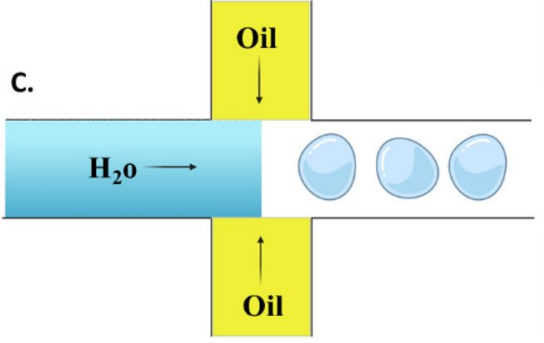

Flow focusing
D.

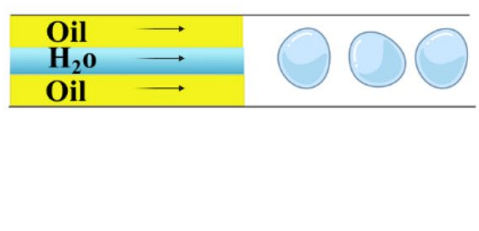

Co-flowing

Fig. 2 Schematic representation of a droplet-based microfluidics system. A Basic design and passive methods of droplet formation, B crossflowing, C flow focusing, $\mathbf{D}$ co-flowing 
for the formation of droplets. These include active flow method and passive flow method (Teh et al. 2008). Passive flow method is widely used for the droplet formation as it does not depend upon an external energy and could produce results with a simplified device (Mashaghi et al. 2016; Seemann et al. 2011; Teh et al. 2008). The active method may include electric, magnetic and centrifugal factors, whereas a passive method would include cross-flowing (T-junction) (Garstecki et al. 2006), flow focusing (Fu et al. 2012; Xu et al. 2009) and co-flowing droplet formation (Zhu et al. 2016) as illustrated in Fig. 2B-D. Droplet formation operates under a low Reynolds number to ensure laminar flow of the liquid. Surfactants are often used to stabilize such microdroplets by decreasing the interfacial tension among the two distinct phases (Baret 2012).

\subsection{Microfluidic chip integrated with 3D culture technique}

Most of the conventional cell array techniques are of a 2D cell culture system that failed to replicate the in vivo microenvironment of any cell or tissue. To accurately study the proper growth of cells, their migration, and pattern, a 3D cell culture technique is mostly preferred (Matsusaki et al. 2014; Van Duinen et al. 2015). To provide accurate information on the in vivo study parameters, microfluidic chips are integrated with the 3D cell culture technique. This may provide a 3D tissue model for drug-screening purposes (Matsusaki et al. 2014). Currently, microfluidic devices are used in in vitro studies to test anticancer drugs as these devices enable the formation of tumor spheroids. They may also control the uniformity of the size of the tumor spheroids (Jeong et al. 2016). Xu et al., introduced a design of microfluidic chip integrated with 3D co-culture for drug sensitivity testing of anticancer drugs. They had cultured lung cancer tissues in a 3D culture which accurately replicated the tumor microenvironment system. Anticancer medicaments were later introduced according to gradient concentration generator
(CGG). The system is also enabled to screen the sensitivities of various anticancer medicaments that were tested (Xu et al. 2013). The microfluidic 3D co-culture chip is designed and fabricated with polydimethylsiloxane (PDMS) polymer. Microchannels are engraved by a photolithography process. The chip is connected to the injection pump to manipulate the progression of the liquid inside the chip. The chip consists of a reservoir, a concentration-gradient generator, and three chambers of cell culture. The CGG comprises an upstream drug input arrangement from where the drug is inserted and a downstream medium input from where the medium is injected, which is further attached to the cell culture chamber. Both the inputs are mixed at the mixing microchannel (light blue, as shown in Fig. 3) to maintain an equal flow rate. With the assistance of this design, dissimilar cells and drugs could be cultured and tested for any disease condition (Bruzewicz et al. 2008; Xu et al. 2013).

\subsection{Microfluidic hydrogel chip}

For the planning of microfluidic chips, PDMS polymer is usually utilized, in light of the fact that it is biocompatible, transparent, profoundly precise, and creates a high throughput. Apart from these qualities, one challenge with the PDMS is that they seldom facilitate cell adhesion or attachment (McDonald et al. 2000). It is known that in cell-biomaterial studies, cell attachment is the initial step. It plays a crucial role in the cellular processes such as cell proliferation and cell differentiation (Leclerc et al. 2003). To overcome this challenge, microfluidic channels could be coated with a cell-compatible hydrogel layer inside a PDMS-layered device (Rosser et al. 2015). Hydrogels are usually composed of a 3D network made up of natural or synthetic polymers that mimic the microenvironment of the extracellular matrix. Natural polymers include agarose, alginate, collagen, dextran, fibrin, and laminin, whereas synthetic polymers include polyethylene glycol (PEG) and PEG diacrylate (PEG-DA). Among these, PEG and its by-products are frequently used
Fig. 3 A schematic design of a microfluidic 3D co-culture chip for cell culture and medication testing (Xu et al. 2013)

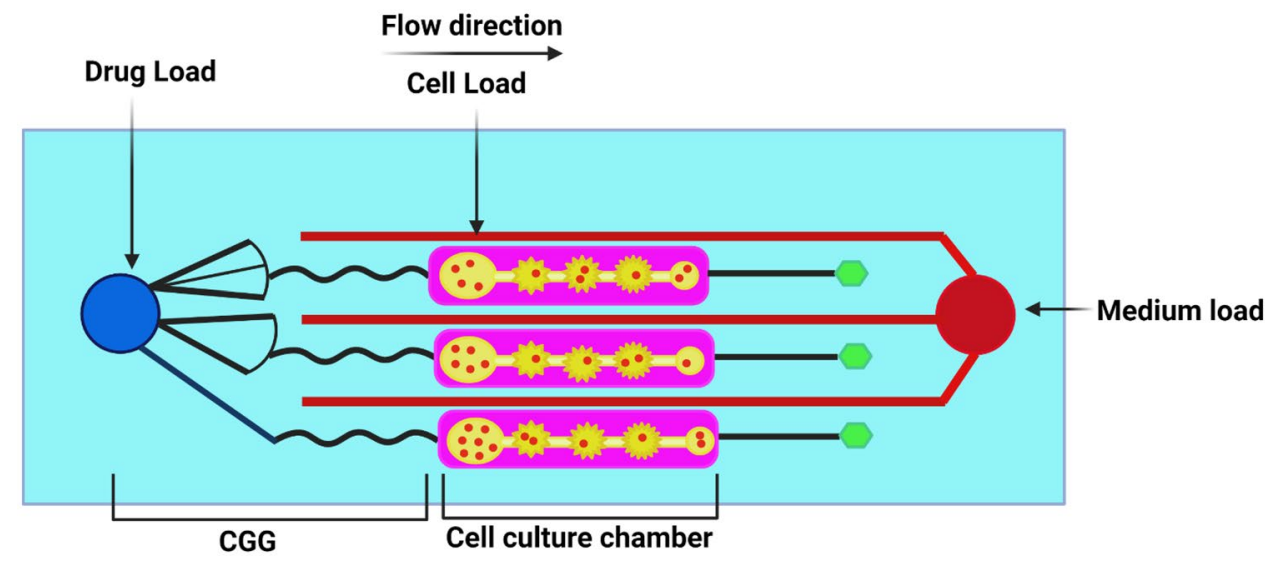


for microfluidic hydrogel devices. Hydrogels are mainly used for the encapsulation of cells/drugs and also for cellular barrier establishment (Beebe et al. 2000).

These 3D hydrogel-based microfluidic chips have been found very helpful in angiogenesis and cell migration. For instance, Shin et al., developed microfluidic systems that was fabricated in polydimethylsiloxane (PDMS) using standard soft lithographic methods. It was comprised of hydrogelincorporating chambers between surface-accessible microchannels to study angiogenesis using cell culture assay. The platform was composed of four gel regions and three channels, each of which is individually accessible. The cells were cultured in the center channel, and the other two side channels acted as control channel and condition channel, respectively. The interior of the channels was coated with extracellular matrix (ECM) proteins to promote cell adhesion. Further, the coating with poly-D-lysine (PDL) hydrobromide was done to promote 3D capillary morphogenesis into the hydrogel. This platform has allowed application of biochemical and biophysical stimuli to multiple cell types interacting over distances of less than one millimeter, thereby replicating many aspects of the in vivo microenvironment. Further, it provided the possibilities for timedependent manipulation of flow and concentration gradients as well as generation of high-resolution real-time images for observing spatial-temporal single-cell behavior, cell-cell communication, cell-matrix interactions and cell population dynamics. This platform has provided a scope for performing heterotypic cell type assays that can be used to study cell survival, proliferation, migration, morphogenesis and differentiation under controlled conditions (Shin et al. 2012).

In another study, Annabi et al., developed hydrogelcoated microfluidic channels for cardiomyocytes culture where they had synthesized photo-crosslinkable gelatin plus tropoelastin-based hydrogel mixture for the purpose of coating (Annabi et al. 2013). Tropoelastin is a soluble precursor to elastin which helps to retrieve the original shape of any tissue (Vrhovski and Weiss 1998). The primary cardiomyocytes cultured on tropoelastin hydrogel layers showed better cell attachment and high voluntary beating rates in contrast with photo-crosslinkable gelatin. This shows that tropoelastin hydrogels may be suitable for the fabrication of $\mathrm{OoC}$, as it provides better elasticity and better exposure for the production of elastic tissues like blood vessels (Annabi et al. 2013; Bakooshli et al. 2013). The microfluidic hydrogel device included an inlet, an outlet and an inside layer of the microchannels where the hydrogels were coated. The microchannels were engraved on the PDMS layer. There are different types of hydrogels, namely linear, T-shaped and branched. The green-shaded area has transport properties in a penetrable hydrogel (Koo and Velev 2017).

Nie et al. developed Vessel-on-a-chip with hydrogelbased microfluidics that was equipped with internal microchannels to offer more in vivo-relevant models for construction of tissues and organs in vitro. The chips were designed by combining the casting and bonding processes with twice cross-linking strategy to obtain a bonding interface that had the same strength with the hydrogel bulk, which was applied to arbitrary combinations of hydrogels. The developed chips show that combination of gelatin and gelatin methacrylate (GelMA) were biocompatible and promoted cell functionalization. The chip offered a promising model for studying vascularization, vascular inflammation, tissue engineering, and drug development (Nie et al. 2018).

It has been observed that hydrogel-based microfluidics chips are more biologically relevant than the PDMS chips. However, the inherited swelling of hydrogels leads to the decrease in mechanical performance and deformation of the structure of chips, which causes difficulty in application processing. To address these issues, Shen et al., non-swelling hydrogel-based microchips by fabricating by covalently cross-linking of the biocompatible copolymer of di-acrylated Pluronic F127 (F127-DA). The non-swelling property of this chip helped in maintaining the as-prepared mechanical strength and channel morphology when equilibrated in aqueous solution at $37{ }^{\circ} \mathrm{C}$. In addition, these chips showed slow degradation rate (within 21 days of incubation) upon conducting the stability studies. Furthermore, using this technology, a vessel-on-a-chip is established that was designed via seeding human umbilical vein endothelial cells (HUVECs) on the microchannel surfaces inside the microfluidic that showed resistance to fluid shear stress and expressed higher endothelial functions than the corresponding static culture (Shen et al. 2019). The diagrammatic representation of swellable and non-swellable hydrogel-based microfluidic chips are shown in Fig. 4.

\subsection{Microfluidic chip merged with detection instruments}

Microfluidic chips could be integrated with location-based instruments to improve effectiveness, speed of the test, and throughput as well as to minimize the expenses and test utilization of the examination (Cui and Wang 2019; Gao et al. 2013). In recent years, there has been an increased interest in the miniaturization of instruments in analytical chemistry. Microfluidic devices are being tested by coupling with different analytical instrumentation techniques, namely mass spectrometry (Wang et al. 2015), polymerase chain reaction-based instrumentation (Ottesen et al. 2006), gas chromatography-mass spectrometry (Pang et al. 2015) and liquid chromatography (Grinias and Kennedy 2016). 


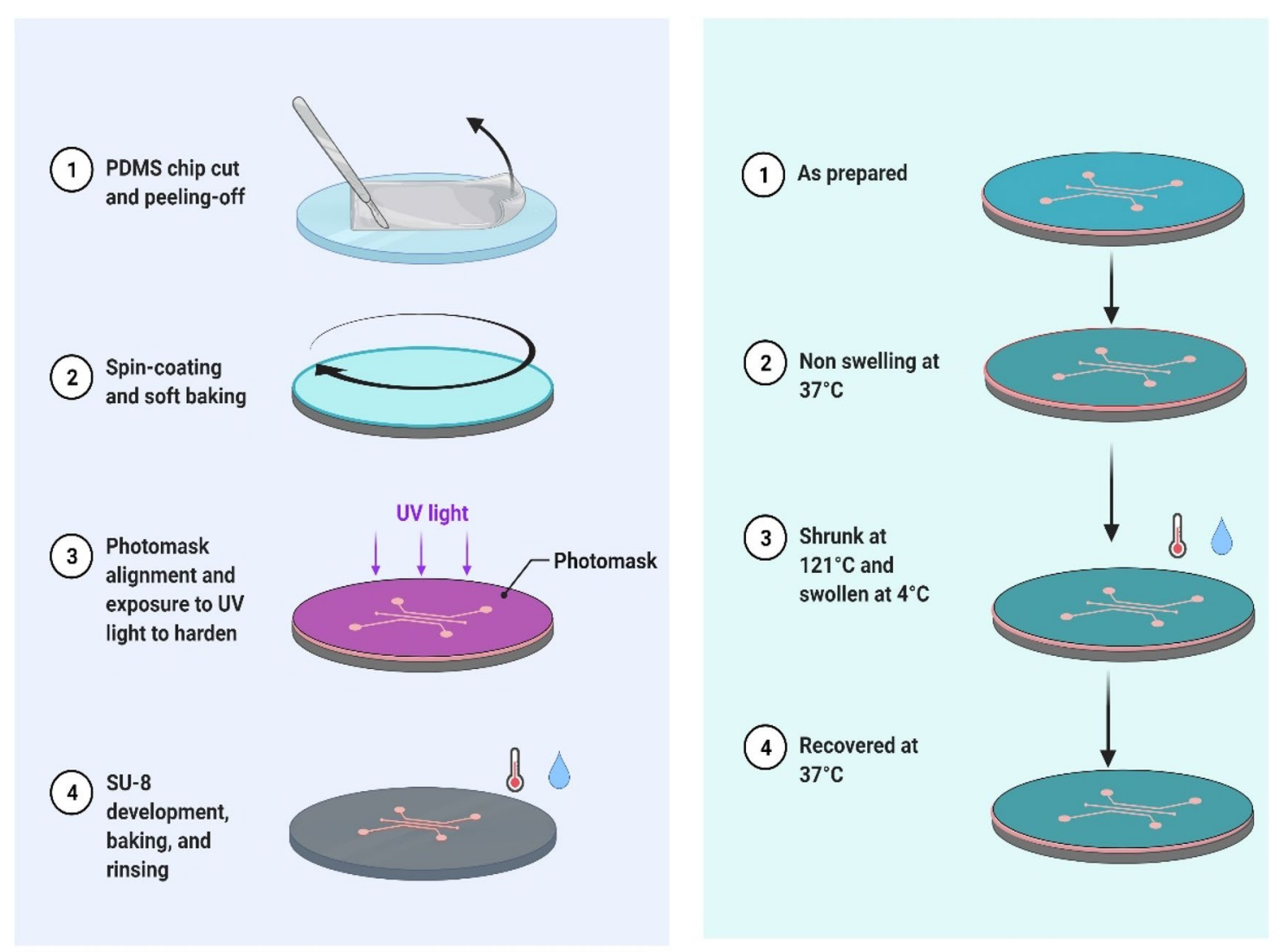

(A) Swelling based hydrogel microfluidic chip

(B) Non-swelling based hydrogel microfluidic chip

Fig. 4 Diagrammatic representation of $\mathbf{A}$ swellable and $\mathbf{B}$ non-swellable hydrogel microfluidic chip

\subsubsection{Microfluidics combined with electrospray ionization and mass spectroscopy technique}

Recently, there has been an increased utilization of microfluidic chips coupled with electrospray ionizers and mass spectrometers (ESI-MS) for high sensitivity and throughput. These systems are equipped to measure samples to picogram range. Apart from the advantages, one significant challenge in the coupling of microfluidics to electrospray ionizer is to improve steady and successful interfaces (Lee et al. 2009a; b). Coupling a microfluidic device to an electrospray ionizer is carried out through several methods that have been employed currently. The primary strategy was to utilize monolithic technique, where electrospray would be executed in a straightforward manner from the microfluidic channels that are opened at the edge of a chip. Disregarding that such kind of technique is straightforward, the major hurdle associated with it includes difficulty to control electrospray ionizer along the chip edge (Lee et al. 2009a; b; Wang et al. 2015). To overcome this challenge, intertwined silica capillaries are embedded toward the ends of the channels to fill in as the electrospray tip. However, such a methodology being troublesome, a third approach was explored where integrated emitters were being used in the designing process monolithically (Gao et al. 2013; Lee et al. 2009a, b).

As per the requirements, single or multiple electrospray emitters are fabricated with different materials, namely PDMS, glass, silicon, quartz, PMMA and polycarbonate (Feng et al. 2015). Different fabrication technologies used include: (a) cleanroom intensive approaches that include $\mathrm{X}$-ray photolithography, HF etching, UV exposure/SU-8; (b) non-cleanroom approaches, namely hot embossing, plasma etching, injection molding and embossing (Feng et al. 2015; Sung et al. 2005). In electrospray ionization mass spectrometry, the sample solution is sprayed through a high-voltage potential capillary by nebulization gas. The sprayed droplets are ionized due to the high-voltage potential in the capillary. Heat desolvation gas evaporates the solvent and its resultant molecular ions are formed (Xue et al. 1997). Electrospray ionization process is primarily employed in the ionization of proteins, peptides, lipids and oligosaccharides (Koster and Verpoorte 2007). The design of the microchip comprises nine microchannels and each of them has two inlet wells. One well is for the sample inlet which can be associated with the syringe siphon. The subsequent well contains a support that can be associated with a high-voltage source. The leave 
ports of the chip are lined up with the orifice of the mass spectrometer utilizing a 3D translational stage as shown in Fig. 5 (Gao et al. 2013; Koster and Verpoorte 2007; Lee et al. 2009a, b; Xue et al. 1997).

\subsubsection{Microfluidics coupled with matrix-assisted laser desorption ionization (MALDI)}

MALDI is one of the common ionization techniques in mass spectrometry, which could be coupled with microfluidic technology for better sensitivity, speed, throughput and cost reduction (DeVoe and Lee 2006). This is because microfluidics has the ability to manipulate small sample quantities with minimal sample consumption, minimal cross-contamination, and high accuracy. MALDI is essentially employed for bigger particles such as proteins, peptides, and different biomolecules (DeVoe and Lee 2006; Wheeler et al. 2005). In this technique, a pulsed laser beam is guided to collide with the sample-matrix mixture. This would result in the conversion of the sample into ions due to translational energy (Kussmann and Roepstorff 2000). Coupling of the MALDI technique with microfluidics may be performed in two ways: on-line or off-line formats (Lee et al. 2009a; b). In the online design, the analyte is conveyed into the mass spectrometer basically by three methodologies, for example, vaporized particles, vessels, or by mechanical methods. However, a major challenge in on-line coupling is that MALDI requires vacuum, whereas the microfluidic operations are done at atmospheric pressure (Chatterjee et al. 2010). Therefore, the off-line coupling is widely used because samples can be directly deposited on the target for subsequent analysis. Sample deposition is accomplished with a robotic target spotter. Both, the matrix as well as sample are mixed on-line before being spotted on a matrix-coated target (Chatterjee et al. 2010; Gao et al. 2013). A plan of the microfluidic chip integrated with the MALDI procedure comprises a T-junction droplet microfluidic type where the drops are framed after the connection between the oil and fluid stage. At that point, the droplets are coordinated from a microfluidic T-junction onto the MALDI plate mounted on a motorized $x-y$ stage by a capillary. For capturing the aqueous droplets, MALDI plate is precoated with teflon and hydrophilic spot regions. The solvents are then permitted to dissipate. 2,5-Dihydroxybenzoic acid (DHB), which is a MALDI matrix, is applied to the dried droplets as shown in Fig. 6 (Feng et al. 2019; Kussmann and Roepstorff 2000; Küster et al. 2013; Wang et al. 2015). This technique is mainly used for bioanalysis reactions such as enzymatic reaction, proteins and peptide analysis.

\subsubsection{Microfluidics combined with PCR technique}

PCR technique is widely utilized in molecular biology for the amplification of DNA strands (Zhang et al. 2006). The amplification process consists of three steps such as, denaturation, annealing, and extension. In the denaturation cycle, the double-stranded DNA loosens up into two single strands at $95{ }^{\circ} \mathrm{C}$. The temperature is then lowered to $55^{\circ} \mathrm{C}$, so that
Fig. 5 Schematic outline of a microfluidic chip electrospray ionizer interface showing electrospray ionization-mass spectrometer (Xue et al. 1997). Copyright 1997, American Chemical Society

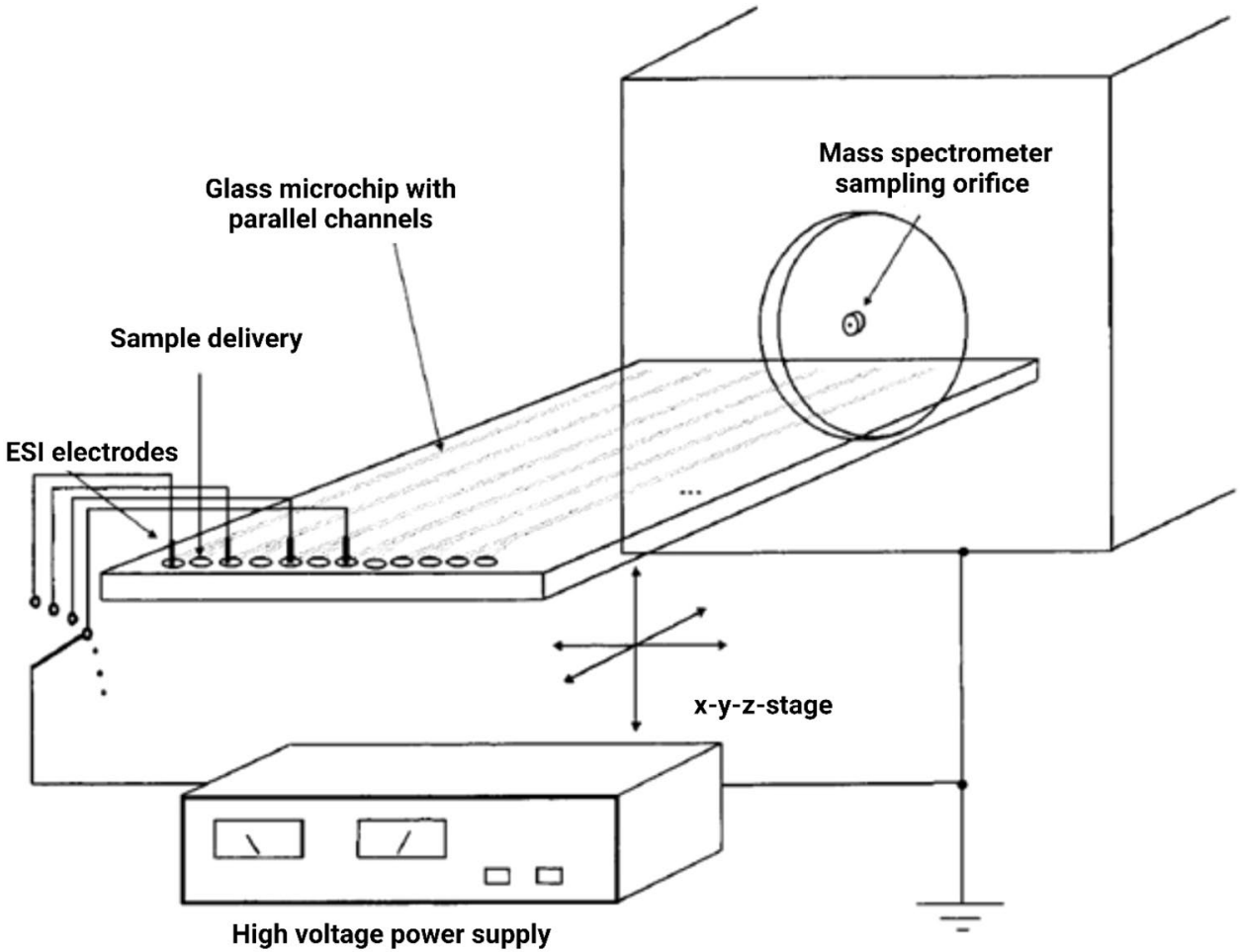




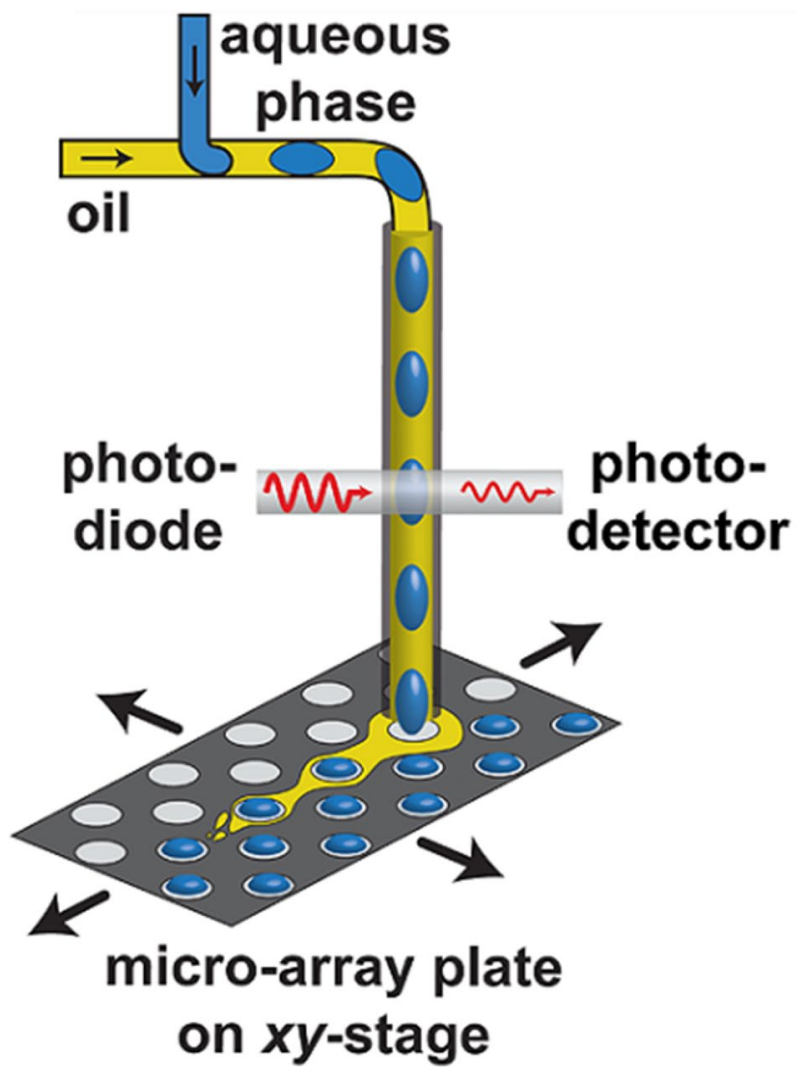

Fig. 6 A schematic outline of the droplet microfluidic strategy used to frame and convey watery droplets to MALDI plate (Küster et al. 2013). Copyright 2013, American Chemical Society the primers could be attached to the DNA strands for annealing. After this step, a polymerase enzyme is attached to the complex. At that particular point, the temperature is elevated to $72{ }^{\circ} \mathrm{C}$ for the extension cycle (Ottesen et al. 2006; Zhang et al. 2006). In the PCR procedure, there are several warming and cooling steps which relatively consume time. However, the absolute analysis time can be reduced by microfabricating the device. Furthermore, the decrease in size prompts a higher surface region where mass and heat transport could be expanded (Ahrberg et al. 2016). In a microfluidic device, flow of the sample is regulated and controlled by syringe pumps. However, employing these pumps would make the operation complex. Therefore, to avoid the use of external pumps, self-propelled continuous-flow PCR is combined with a capillary-driven microfluidic chip for the amplification of DNA strands. In this, after pouring the PCR solution, it is simply transferred autonomously via capillary forces and amplification of DNA strands takes place as shown in Fig. 7 (Park et al. 2011; Tachibana et al. 2015).

\subsubsection{Microfluidics integrated with liquid chromatography (LC)}

Liquid chromatography is a separation technique which is utilized to isolate particles or ions that break down in a dissolvable solvent. It is preferred for the separation of complex mixtures of proteins, peptides and drug metabolites (Snyder et al. 2011). In the usual analysis of macromolecules such as proteins or peptides, there may be some challenges such as complexity, wide range of concentration, differently expressed proteins during cell development, low-level
Fig. 7 Design of a PCR system combined with microfluidic chip (Tachibana et al. 2015). Copyright 2015, Elsevier

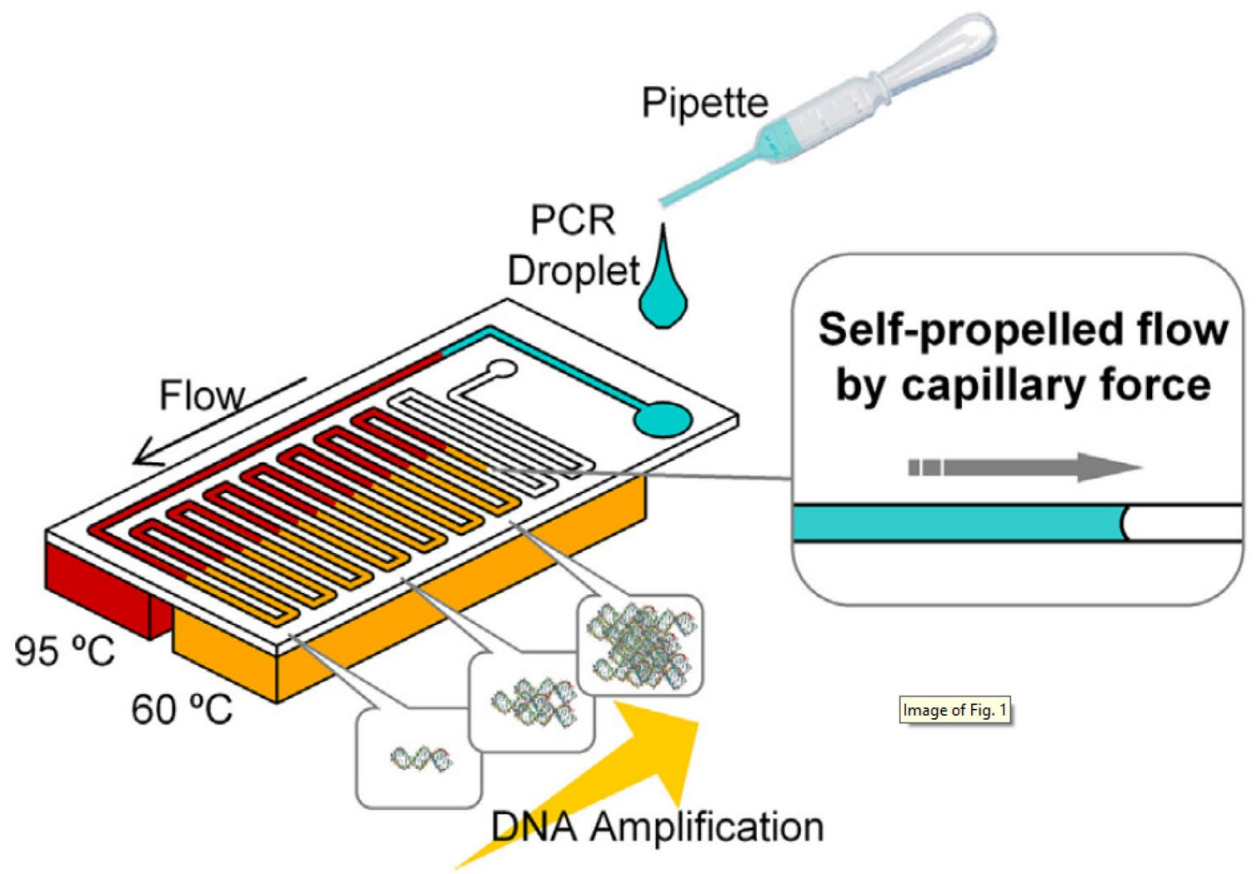


expression for certain components, sample preparation at microlevel and availability (Akshatha and Gurupadayya 2018; Snyder et al. 2011). To overcome these difficulties, multiplexed diagnostic stages are being built up which allow high throughput and precise outcomes. Miniaturization of a device enables small sample manipulations (0.1-1 nL) which results in short analysis time and simultaneously reduces the cost (Grinias and Kennedy 2016). Completion of various stages, namely sample infusion, division, labeling, and detection should be possible in fewer minutes. It can also perform exact and precise sample dealing operations. Due to these advantages, microfluidic technology is combined with LC for the biomolecule analysis (Akshatha and Gurupadayya 2018; Huft et al. 2013). Microfluidic LC devices are fabricated by photolithography and wet-chemical etching. The device typically consists of pumping channels, eluent inlet, outlet reservoirs, separation channels, double T-junction containing sample plug, sample reservoir, sample inlet and outlet, sample waste reservoir, electrospray capillary emitter, and waste reservoir as shown in Fig. 8 (Lazar et al. 2006).

\subsubsection{Microfluidics coupled with gas chromatography- mass spectrometry (GC-MS)}

GC-MS is a separation technique that assists in the investigation of modest and unstable compounds such as hydrocarbons, essential oils, and basic molecules such as steroids, hormones, and unsaturated fats. It can likewise be utilized for the investigation of fluid, solid, and gas samples (Adams 2007). It could also recognize complex combinations, to evaluate analytes, and trace level chemicals in organic contamination. In GC-MS, the sample is usually rendered volatile because of which it effectively disintegrates, and different segments are isolated with the support of a capillary segment packed with the stationary stage (Stein 1999). A carrier gas such as helium, argon, or nitrogen propels the components of the sample, and after getting separated, they elute from the column at different retention times. After elution, the segments get ionized by mass spectrometer utilizing an ionization procedure, and the particles get isolated through the mass analyzer depending on the respective mass-to-charge $(\mathrm{m} / \mathrm{z})$ proportions (Adams 2007; Willard et al. 1988). A GC-MS microfluidic chip is designed for the determination of ambient gaseous molecules. The device comprises a micro-reactor, gas, fluid splitting and combining channels, mixing junctions and microchannels as shown in Fig. 9. The micro-reactor is used for three purposes: as a mixer and reactor, as a heater, and as a pre-concentrator. The microchannels are engraved by hydrofluoric acid etching (HF etching) which is a form of wet etching and thermal bonding process on the glass microfluidic chip (Pang and Lewis 2012; Pang et al. 2013).

\section{Biomedical applications of microfluidic technology}

Microfluidic technology has various applications in disciplines of drug discovery (Dittrich and Manz 2006), proteomics (Mouradian 2002), drug screening (Damiati et al. 2018), medical diagnostics, biosensors (Lee et al. 2010; Rivet et al. 2011) and tissue engineering (Andersson and Van Den Berg 2004). Moreover, in the field of pharmaceutical analysis, microfluidic chip technology plays a significant role in drug development (Weigl et al. 2003), analysis, detection of pesticide residue and in food safety sensing, where the chip is integrated with various other equipment's, namely colorimeter, fluorimeter and spectrophotometer (Gao et al. 2020). It further assists in the monitoring of hormone secretion (Roper et al. 2003), peptide analysis integrated with HPLC (Yin et al. 2005), analysis of tumor cell metabolism (Chen et al. 2012), and several other applications.

At a drug discovery level, it mainly emphasizes on identification, characterization, purification and structure elucidation of chemical moieties (Pihl et al. 2005). It is well
Fig. 8 Schematic portrayal of LC microfluidic chip framework which incorporates 1. siphoning channel; $2 \mathrm{~A}$ and $2 \mathrm{~B}$. eluent inlet reservoir; 3. eluent source repository; 4. double T-junction containing sample plug; 5 . division channel; 6. sample reservoir; 7. sample squander channel; 8. sample inlet channel; 9. sample outlet channel; 10. electrospray capillary emitter and 11. LC squander reservoir (Lazar et al. 2006) Copyright 2006, American Chemical Society

\section{A. Loading of sample}

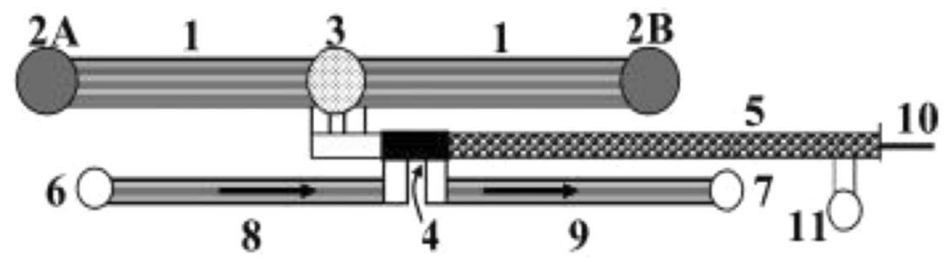
B. Analysis of sample


Fig. 9 Basic design of a microreactor with mixing junction, gas and fluid splitting and combining channels along with microchannels

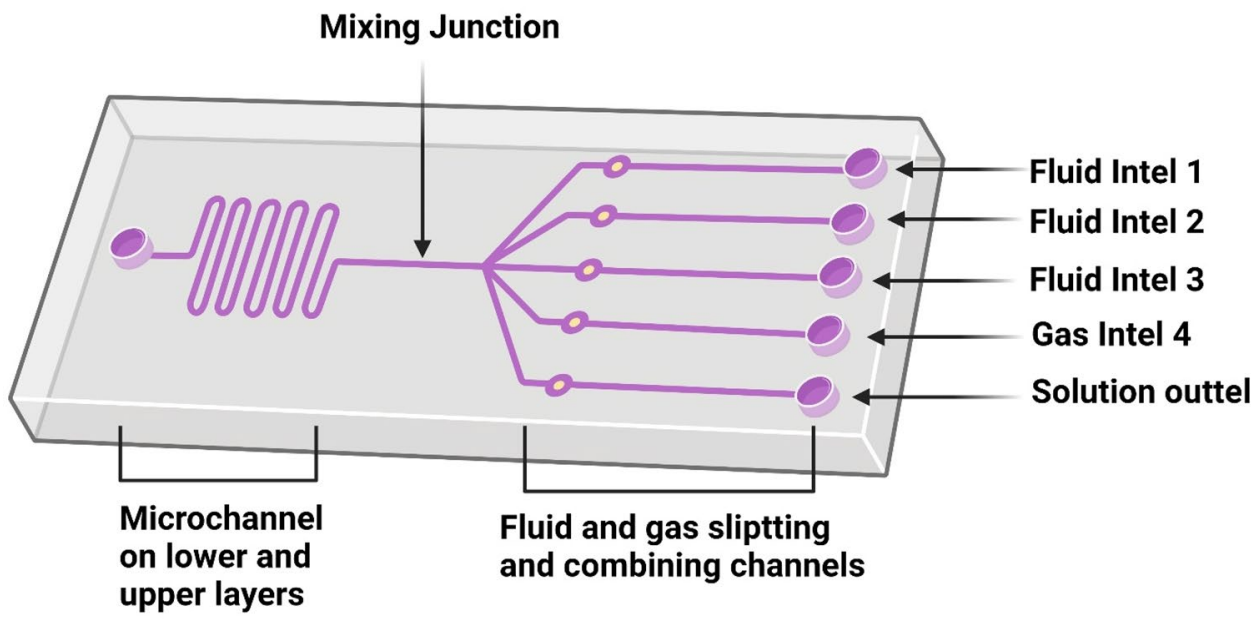

reported that, during the process of analysis, there are several significant challenges that may hamper the results, namely low throughput, the need for a larger quantity of sample or reagent, reduced accuracy and tediousness in the process. In such cases, microfluidics chip technology is employed to minimize these challenges. Multiple microchannels permit high throughput which further demonstrates cost-effectiveness and rapid increase in the rate of how drugs are screened (Kang et al. 2008; Warkiani et al. 2014; .

We attempt here to establish the recent advances in the designing of different types of microfluidic chips along with their roles and applications in the field of biomedical sciences. Microfluidic chip technology is primarily applied for therapeutic and diagnostic purposes. It is basically classified on the basis of device configuration/setup and device application. In biomedical field, various types of microfluidic chips are currently being used for different purposes such as lung-on-chip, kidney-on-chip, skin-on-chip, brain-on-chip, and gut-on-chip. 'Organ-on-chip' technology helps to simulate the physiology of organs, namely lung, kidney, heart, gut, and brain to name a few. Moreover, it further extends its scope in targeted drug delivery systems using 'droplet microfluidics'. In the analysis of a single cell, droplet microfluidics is extensively used because of its ability to isolate the cells. Microfluidic technology is also integrated with detection instruments and equipment's for better sensitivity, speed, and throughput.

\subsection{Droplet-based microfluidics chips}

Maher et al., reported use of microfluidic chip in treatment of colorectal cancer where multifunctional microspherical magnetic and $\mathrm{pH}$-responsive transporters were utilized. These were designed by droplet-based microfluidics. For colorectal cancer therapy, $\mathrm{pH}$ stimuli-responsive drug delivery platform played an important role here. The microfluidic chip was engineered with $\mathrm{pH}$-sensitive magnetic microspherical carriers constituting a synergistic treatment for colorectal cancer. Anticancer drugs such as 5-fluorouracil and curcumin were selected because their combination had shown a synergistic effect on colorectal cancer treatment. In this study, the chemotherapeutics were stacked on permeable silicon nanoparticles and magnetic bacterial iron oxide nanowires. After the stacking, the medications were exemplified into polymeric microspheres utilizing a droplet-based microfluidic. For the controlled drug delivery, the microspheres were prepared with hypromellose acetic acid derived from a succinate polymer. This particular polymer has a unique property whereby it was insoluble in the acidic $\mathrm{pH}$ of the stomach and got solubilized at the $\mathrm{pH}$ of colon and rectum thereby releasing the drugs. The polymeric microspheres showed a thin size distribution. The synergistic activity of chemotherapeutics-stacked microspheres couple microchips were found very effective in treating cancer (Maher et al. 2017).

\subsection{Organ-on-chip (OoC)}

OoC is a micro-engineered 3D in vitro tissue model where micro-compartments are connected by several microfluidic channels. It helps in replicating the physiological environment of any organ (Cui and Wang 2019; Wu et al. 2020). Moreover, it may also be used for biochemical analysis. In the drug discovery process, it is important to predict the effects of any drug before it could be subjected to clinical trials. This step is usually time consuming and expensive. In contrast, OoC mimics the whole physiological part of any organ in a simplified way using the microfabrication technique (Zhang et al. 2018a; b). It reduces cost and increases throughput by reducing the gap between preclinical testing and human trials. Franzen et al. dealt with this and estimated that a decrease of $10-26 \%$ in R\&D costs per new drug, and thus, it shows a positive cost impact (Franzen et al. 2019). This type of chip has several advantages, namely the 
compartments inside the microfluidic device enhance the control of microenvironment, precise control of the physical conditions and effective manipulation of the communication between the distinct tissues. It can also produce nutrients and oxygen for providing supply to the organs along with the elimination of catabolic metabolites (Kimura et al. 2018). The application of OoC may be limited to purely surface effects, namely the drug product being adsorbed on to the inner lining, and second, the laminar flow that may exhibit relatively a lesser degree of mixing (Probst et al. 2018). OoC may be of different types, namely brain-on-chip, heart-onchip, liver-on-chip, kidney-on-chip, and lung-on-chip. The different types of $\mathrm{OoC}$ are illustrated schematically in Fig. 10.

\subsubsection{Brain-on-chip (BoC)}

It is well known that factors directly related to neurons and cell-cell interactions play an important role in case of brain tissue functioning. The study of the brain and its tissues are largely complex, which renders 2D models such as Petri dish or culture flasks ineffective, as these systems fail to simulate the actual physiological environment of the brain (Bang et al. 2019). To overcome this limitation, researchers currently are working on the possible development of brain-on-a-chip platform where the physiological factors of the brain could be studied under an advanced miniaturized-engineered platform that can be prepared by multistep lithography technique (Fig. 10A). It has further enabled the study of brain tissues by fabricating different dimensions of microchannels (Jahromi et al. 2019). Another study which employed an in vitro cell culture technique was previously reported, where the axons and soma were physically separated, thereby allowing only the axons to transit through the microchannels. With the help of this technique, neuroscientists could study the features of the axon itself, or may determine the mechanistic action of drugs on the axonal part, and could analyze axonal regeneration after axotomy. It is note-worthy to mention that microchannels may cause shear stress on the tissues or cells resulting in cellular damage. Air bubbles trapped under the microchannels may disrupt the flow properties and may cause cellular damage (Harrison et al. 1907). A sandwich design is usually preferred in the designing of such 3D devices, where the endothelial cells are grown in the upper layer and the brain cells are grown in the lower layer, bifurcated by a porous membrane which acts as blood-brain barrier (BBB).

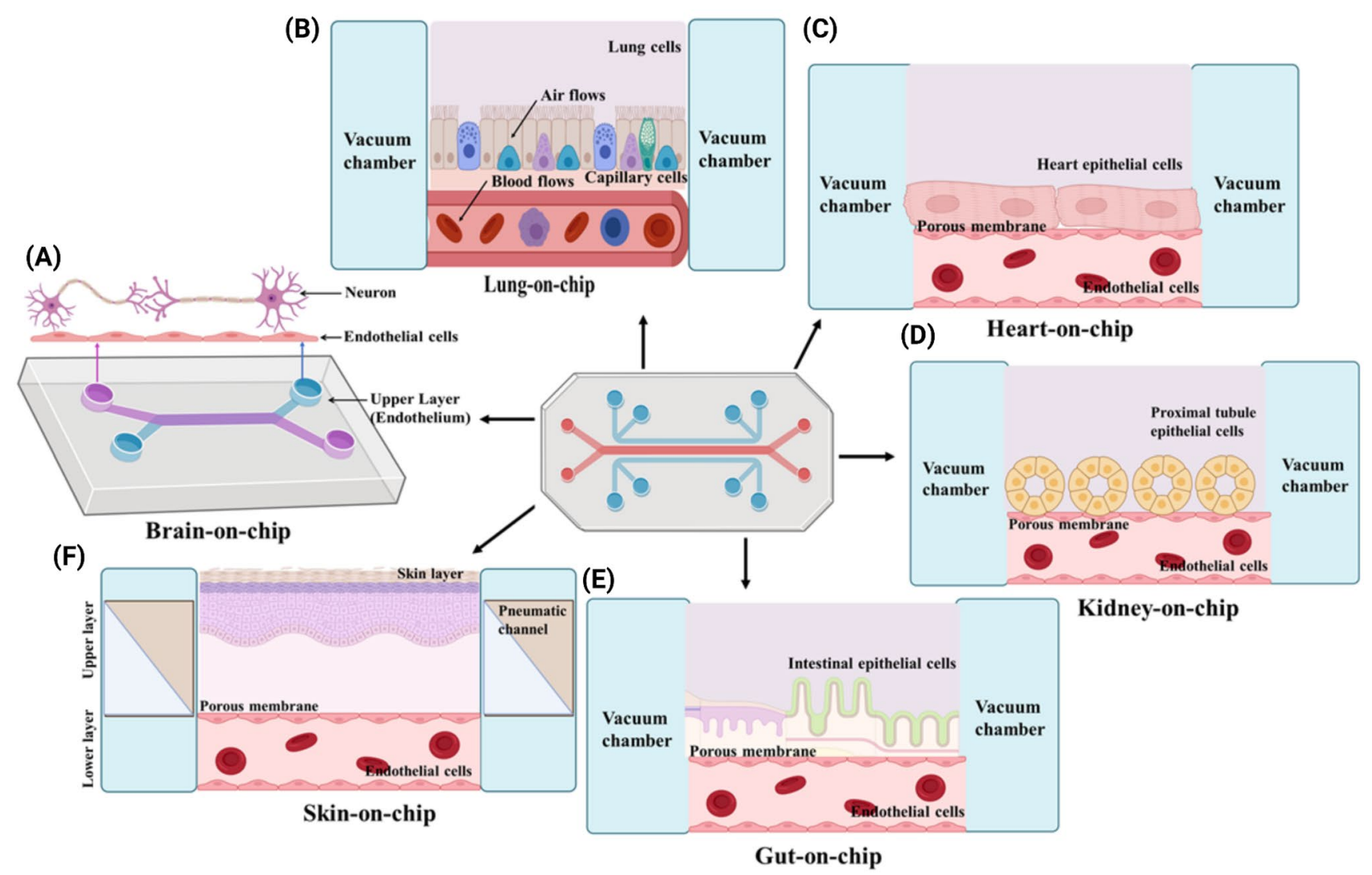

Fig. 10 Various types of organ-on-chip (OoC) systems 


\subsubsection{Lung-on-chip (LoC)}

Lung-on-chip (Fig. 10B) is another 3D engineered complex model of a living human lung on a microminiaturized device. It essentially constitutes a human lung and blood vessels. This system may assist largely in the physiological study of the lungs. Moreover, it also helps in studying the features of the nanoparticles absorbed in the alveoli sacs and further mimics the inflammatory response triggered by pathogens. Furthermore, it can be used to test the effects that are caused by environmental toxins, and aerosol products (Shrestha et al. 2020). LoC enables researchers to study the in vitro physiological actions of the organs or human body, and hence, it is being employed in the strategic implementation of therapeutic modalities in different pulmonary diseases. In tissue designing, microfluidic innovation assumes an essential function in noticing the development of complex tissues by providing oxygen, sustenance, and blood. It develops a microenvironment for the lung cells to study the physiological activities (Huh 2015). Wyss Institute has designed various pulmonary microchips that demonstrate the working of a typical LoC. These microchips further are capable of mimicking pulmonary edema (Ingber 2011). The design of the LoC device includes two layers: the upper layer which contains the pulmonary cells and the lower layer that consists of capillary cells. Furthermore, the two layers are bifurcated by a thin porous membrane.

\subsubsection{Heart-on-chip (HoC)}

Heart-on-chip is an advanced type of OoC which mimics the overall physiology of the human heart after the administration of dosage forms or, a specific drug molecule (Grosberg et al. 2011). Some of the adverse reactions have been observed using this chip. Mathur et al., in 2015 had explained the inadequacy of animal trials to estimate the exact pharmacokinetics and pharmacodynamics of a test drug molecule with respect to the human body. For that, microfluidic chip technology plays a crucial role in the study of cardiovascular diseases, cardio-vascular-related drug development, cardiotoxicity analysis, and also to study cardiac tissue regeneration (Mathur et al. 2015). Sidorov et al., in 2016 had created a I-wired HoC. They detected contractions of the cardiac muscle, which were measured by an inverted optical microscope. Furthermore, engineered 3D cardiac-tissue constructs (ECTCs) now have the ability to replicate the complex physiology of the cardiac tissues under normal and diseased conditions (Sidorov et al. 2017). Figure 10C shows the schematic representation of heart-onchip in which the upper layer consists of heart epithelial cells and lower layer consists of heart endothelial cells. Both the layers are separated by a porous membrane. It also consists of vacuum chamber which helps in pumping of the blood.

\subsubsection{Kidney-on-chip (KoC)}

Limitations of traditional approaches or conventional methods, such as varying or inappropriate cellular functions and physiology rendered pathophysiological study of nephrons inaccurate and error prone. In contrast, integration with microfluidic technology has proven to produce better and precise results. $\mathrm{KoC}$ is essentially prepared by integrating the renal tubular cells with microfluidic chip technology (Kim and Takayama 2015). It is majorly used in assessment of kidney toxicity. Only $2 \%$ of the drug failures are screened during the preclinical stage and around $20 \%$ after the clinical stage. This justifies the use of KoC to study human nephrons on a single micro-sized chip (Wilmer et al. 2016). Lee et al., had previously explained that the pharmacodynamic and pathophysiologic reactions of the nephrons were more pragmatic in microfluidic 3D technology as compared to the $2 \mathrm{D}$ models. $\mathrm{KoC}$ has been developed and demonstrated to show better in vivo consequences of drug nephrotoxicity and the system has been employed further in the determination of various drug-induced biological responses (Lee and Kim 2018). In addition, it also assists in the culture of proximal tubules which are used for the observation of biomarkers that predicts drug-induced kidney injury (DIKI) and drug interactions (Kim and Takayama 2015; Wilmer et al. 2016). A simple design of a kidney-on-chip model basically consists of two layers. An upper layer contains the proximal tubule epithelial cells and a lower layer contains the endothelial cells. A porous membrane situated in the middle separates both the layers as illustrated in Fig. 10D.

\subsubsection{Gut-on-chip (GoC)}

A GoC system mimics the physiology of the human gut. It explains about the major functions of the gut, namely digestion, absorption of nutrients, regulation of enteric nerves, excretion of waste materials from the body, and the pathophysiology of the human gut accompanied by the microbial symbionts. The GoC model is primarily used for the precise reproduction of the in vivo environment of the gut with desired microfluidics parameters (Ashammakhi et al. 2020). Kim et al., studied the peristaltic movements of the intestines when a human GoC was inhabited by the gut microflora (Kim et al. 2012). The microminiaturized device was designed by aligning two microchannels: an upper and a lower, engraved on a PDMS layer which was partitioned by a porous pliable membrane coated with ECM. The setup was enveloped by human intestinal epithelial cells that imitate the physiology of the human gut as shown in Fig. 10E. Such a system could mimic the peristaltic movement of a human gut under certain specific factors, namely flow rate of fluid, minimal shear stress over the microchannels, and 
cyclic strain (Ashammakhi et al. 2020; Grandfils et al. 2020; Kim et al. 2012).

\subsubsection{Skin-on-chip (SoC)}

SoC is a bioengineered model where the skin tissues are cultured within a microfluidic system, that is sufficiently capable to mimic the 3D microenvironment of the natural human skin (Wufuer et al. 2016). To fabricate the microminiaturized SoC model, human skin tissues are integrated onto the microfluidic platform, so that it may mimic the in vivo conditions of the human skin (Zhang et al. 2018a; b). The conventional 2D model fails to recreate the multiplex 3D cell to cell, and cell to matrix interactions found in the body. However, this could be studied with the help of the 3D SoC model. To represent the epidermal and dermal layer, Lee et al. (2009a; b) used 3D-bioprinted keratinocytes and fibroblasts to create human skin tissues. The system typically has predominantly three layers: a lower layer, a middle layer, and an upper layer. The lower layer contains the microvascular channel. The porous membrane is situated in the middle/ intermediate layer which separates the upper and lower layers, whereas the upper layer comprises the culture chamber and the lateral pneumatic channels. The basic setup of a $\mathrm{SoC}$ is illustrated in Fig. 10F. A microvascular channel provides mechanical support for the formation of the endothelial monolayer. Capillary walls formed by the endothelium layer regulate the transport, hemostasis, and inflammatory responses. Pneumatic channels are primarily designed for mechanical stimulation (Lee et al. 2014).

\subsection{Microfluidic chip for single-cell analysis}

Single-cell examination primarily involves the investigation of cell-to-cell associations in their microenvironment at a single-cell level. It incorporates the investigation of genomics and transcriptomics of a single cell (Yin and
Marshall 2012; Yue and Xue-Feng 2006). In living tissues, each cell type has a definite lineage and function. Based on this assumption, in any case, smidgens of proof from various investigations of single cell unveil the fact that cells inside a cell populace are heterogeneous, and are comprised of individual cells that contrast incredibly (Hodzic 2016). Single-cell analysis is employed to study diseases, assists in drug development, and understand physiological functions of individuals. In the field of cell science, DNA sequencing, gene cloning and monoclonal antibody production, the employed PCR techniques have resulted in an increase in the number of samples, reagents, and assays. To beat these challenges, microfluidics has been presented which can play out an assortment of bioassays with negligible amount of samples and limited reagent utilization (Wheeler et al. 2003). A microfluidic network isolates the single cell from the cell suspension. Valves and pumps precisely deliver the volume of the reagents in nanoliters to the cells. The design of a microfluidic chip for single-cell investigation is made on the PDMS layer by multilayer soft lithography method (Unger et al. 2000). In Fig. 11, the dark and light color shows the fluidic and control channels. Reactant inlets are denoted by ' $R$ ' shield buffer (SB) and focusing buffer (FB). Inlets are included to reduce the fluctuation and centering of the fluid. V1-V8 are the valves that operate by applying pressure to manage the inlets. Pumps are activated by the actuation of P1 to P6 in series. After the activation of the pump, the flow rate fluctuations are regulated by the crisscross region of reagent and shield buffer medium. By utilizing hydrostatic weight, the cells are pushed from the cell channel to squander and made to zero at midway by centering support (Hodzic 2016; Kang et al. 2014; Wheeler et al. 2003).

\subsection{Model organism}

Model organisms are species that may be easily manipulated and bred in a typical laboratory setting. They are easy

Fig. 11 Schematic diagram of a single-cell analysis device (Wheeler et al. 2003). Copyright 2003, American Chemical Society

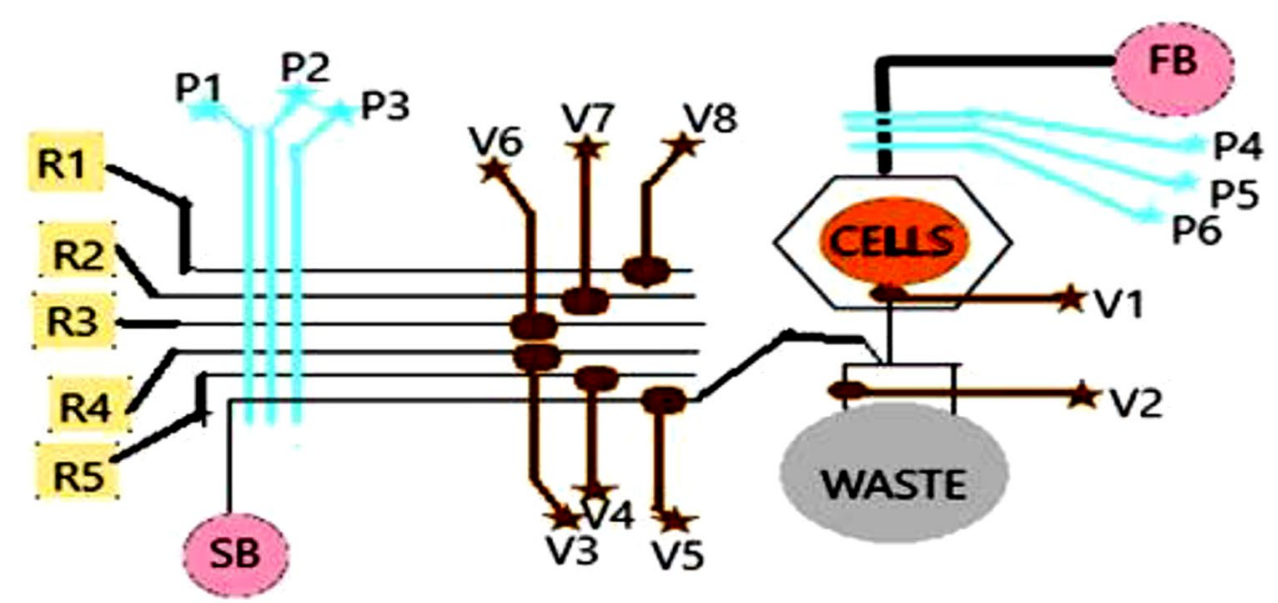


to maintain and assist to understand various biological processes. Some of the widely used model organisms are Mus musculus, Drosophila melanogaster, Caenorhabditis elegans, Saccharomyces cerevisiae, and Danio rerio (Leonelli and Ankeny 2013). C. elegans is a well-reputed model organism for the investigation of neuronal and olfactory responses (Chronis et al. 2007). They are mainly used in genetic research because they can breed on a large scale. In addition, they boast a very short generation time and their genes are similar to humans. Apart from these advantages, there are few challenges such as treatment of multicellular living beings, efficient control of the microenvironment, regulating the controls during phenotype testing, screening, and imaging of model creatures (Crane et al. 2010). Microfluidics may play an important role in overcoming these challenges, especially in the study of model organisms. Microfluidic tools have been employed in the study of the model organisms where they are immobilized by passive immobilization using microchannels. By multilayer soft lithography, the microchannels could be engraved on the PDMS membrane. Droplet-based microfluidic (DBM) technique could be adopted where the eggs/larvae of the model organisms are encapsulated into the droplets and further used for the study purpose (Hwang and Lu 2013). The DBM strategy comprises two locales: a T-junction droplet generator and a droplet trap exhibit. Arrangement of droplets occurs in the droplet generator by shearing a scattered fluid stage with a consistent oil stage. The droplets are then captured in the droplet trap array. Syringe pumps direct and control the pace of the streams in both the stages (Shi et al. 2011). Figure 12 shows a design of a model organism microfluidic chip ( $\mathrm{Ge}$ et al. 2018).

Choudhary et al. designed multi-channel microfluidic perfusion platform for culturing zebrafish embryos and capturing live images of various tissues and organs inside the embryo. They micro-fabricated fish and chips in silicon and glass that was containing three different parts. These include a microfluidic gradient generator, a row of eight fish tanks, and eight output channels. In the fish tank, the fish embryos were individually placed. The fluidic gradient generator platform supported the analysis of drugs and chemicals in a dose-dependent manner with high reproducibility and accuracy. It provided a unique perfusion system that ensured a uniform and constant flow of media to the fish tank with a possibility of efficient waste removal. Embryonic movements were restricted in the fish tank, except for live imaging of internal tissues and organs. To validate the reproducibility of develop chip, valproic acid was taken as model drug and embryonic development of fishes were tested with/ without induction of valproic acid. The results showed that embryos treated with valproic acid showed abnormalities in their development (Choudhury et al. 2012).
Fig. 12 Design of a model organism microfluidic chip (Ge et al. 2018). Copyright 2018, Elsevier

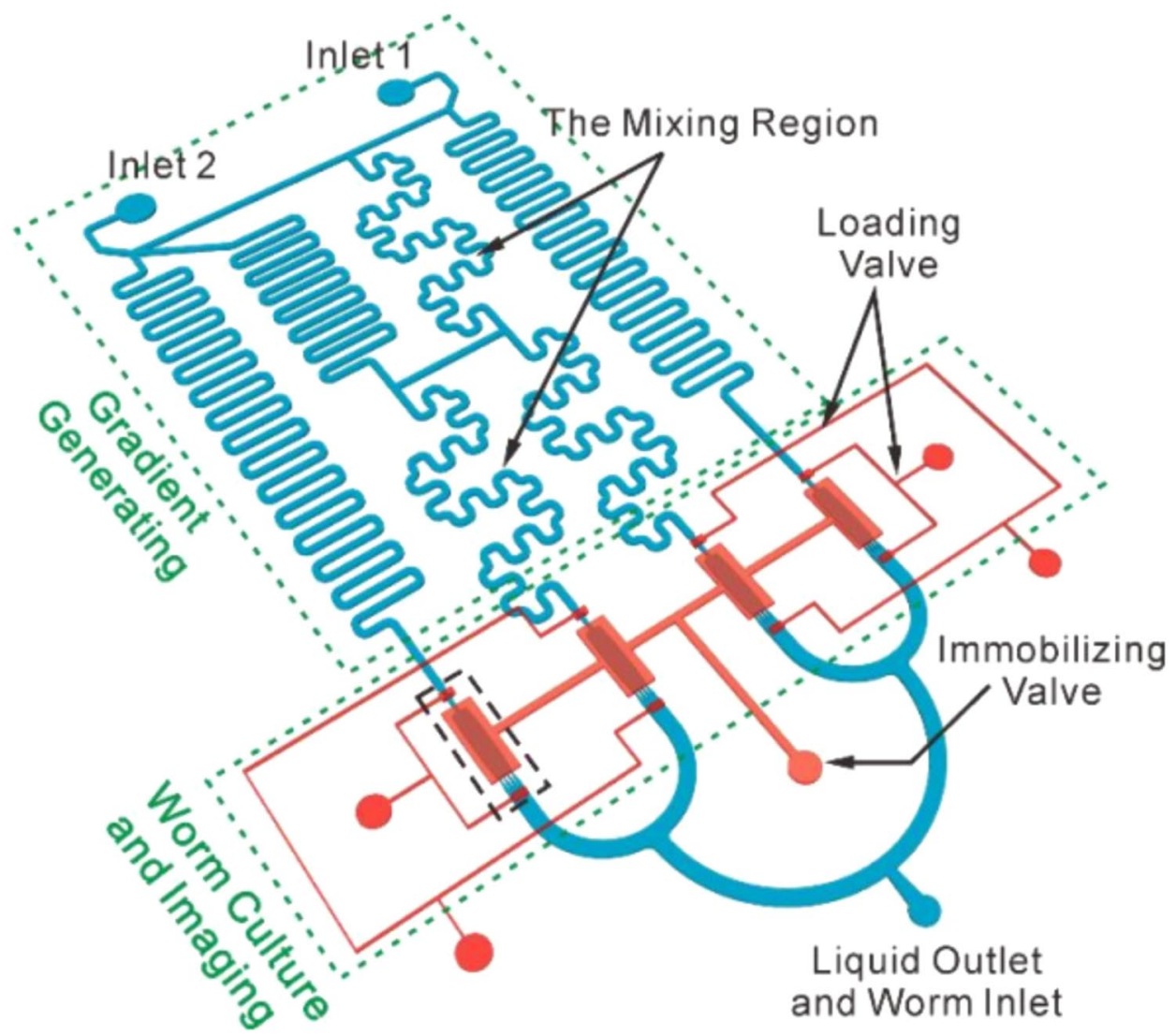


Similarly, Li et al. developed zebrafish on a chip for realtime monitoring of drug-induced developmental toxicity. The microfluidic system has been developed for evaluation of phenotype-based toxic and teratogenic effects of drugs on embryonic development of zebrafish. It was developed using zebrafish (Danio rerio) embryos and larvae as the model organism. The chip consisted of two independent functional units, enabling the assessment of zebrafish embryos and larvae. Each unit was consisting of a fluidic concentrationgradient generator and a row of seven culture chambers to accommodate zebrafish. The accuracy and reproducibility of results obtained using this platform was examined through evaluation of aminophylline induced toxicity and teratogenicity on 210 embryos and 210 larvae (10 individuals per chamber) as model experiment. The quantitative evaluation of effect of aminophylline on zebrafish embryonic development was done by recording a series of physiological indicators such as heart rate, survival rate, body length, and hatch rate. The toxic effect of aminophylline on zebrafish larvae was measured by assessment of clonic convulsion rate in combination with mortality. The results indicated that aminophylline could be able to induce deformity and cardiovascular toxicity in zebrafish embryos as well as their larvae. Hence, this device can be used as tool for the examination of indexes beyond toxicity and teratogenicity at the sub-organ and cellular levels and could also provide a potentially costeffective and rapid pharmaceutical safety assessment tool (Li et al. 2014).

In another research, Chen et al., developed acoustofluidic rotational tweezing platform that enabled contactless, highspeed, 3D multispectral imaging and digital reconstruction of zebrafish larvae for quantitative phenotypic analysis. This platform was reported to achieve contactless and rapid ( $\sim 1 \mathrm{~s} /$ rotation) rotation of zebrafish larvae, which further enabled multispectral imaging of the zebrafish body and internal organs. The authors also claimed that the developed acoustofluidic rotational tweezing system was very helpful for the researchers working in the area of developmental biology, small molecule screening in biochemistry, and preclinical drug development in pharmacology (Chen et al. 2021).

\section{Role of microfluidic chip in therapy and diagnosis}

Microfluidic technology holds a vast range of applications in most fields, namely drug discovery, food technology, electrophoresis, PCR, chemical synthesis, proteomics, tissue engineering, medical diagnosis, single-cell studies, and microarray systems (Cui and Wang 2019; Li and Zhou 2013). Microfluidic devices are miniaturized devices that can control a modest quantity of test sample (like biomolecules, cells, or particles). These microminiaturized devices have the potential for biomedical analysis and diagnosis as they only consume a small volume of samples and reagents which simultaneously reduces the cost of the experiment $(\mathrm{Li}$ and Zhou 2013). Furthermore, they also have the capacity to produce high throughput and accurate results (Cheong et al. 2010; Cui and Wang 2019; Upadhyaya and Selvaganapathy 2010). In the field of biomedical sciences, these chips are mainly used for diagnostic purposes such as in the diagnosis of pathogens, cancer screening, monitoring cardiovascular events, detection of deadly blood infections, genetic analysis, and several other low-cost bioassays (Li and Zhou 2013; Webster et al. 2011). There are various case studies based on therapy and diagnosis where microfluidic technology is employed.

\subsection{Microfluidic technology used in therapy}

In last 30 years, microfluidic chip has emerged out as great tool in the field of cancer therapy for its diagnosis and treatment. Various types of cell and tissue cultures, including $2 \mathrm{D}$ cell culture, 3D cell culture and tissue organoid culture could be performed on microfluidic chips. Patient-derived cancer cells and tissues can be cultured on microfluidic chips in a visible, controllable, and high-throughput manner, which greatly advances the process of personalized medicine. Moreover, the functionality of microfluidic chip is greatly expanding due to the customizable nature (Guo et al. 2021). In addition, it has been found economical as it enables the processing of trace amount of samples such as cells from patient biopsies, provides a high level of automation, and allows the setup of complex models for cancer studies (Mathur et al. 2020). There are various endeavors made in the development of microfluidic chips for their theranostic use. For instance, the microchips have been designed to investigate cancer cascade, which include tumor growth and expansion (Fig. 13), angiogenesis (Fig. 14), progression from early to late stage lesions involving epithelial-mesenchymal transition (EMT), tumor cell invasion (Fig. 15) and metastasis (Fig. 16) (Sontheimer-Phelps et al. 2019).

The tumor growth on-chip models have been designed for identification of neighboring normal parenchymal cells and ECM in the local tissue microenvironment that can influence the growth of various types of cancer. As an example, the co-culture of T47D human breast carcinoma cells with immortalized human mammary fibroblasts (HMFs) and different combinations of various ECM components in a microfluidic device revealed that HMFs promote increased growth of breast cancer cell clusters, especially in fibronectin-rich ECM compared with monocultures (Montanez-Sauri et al. 2013). Furthermore, it also facilitated the analysis of the morphology and growth of T47D cell clusters in response to broad-spectrum inhibitors of matrix metalloproteinases 


\section{A Early-stage primary tumour}

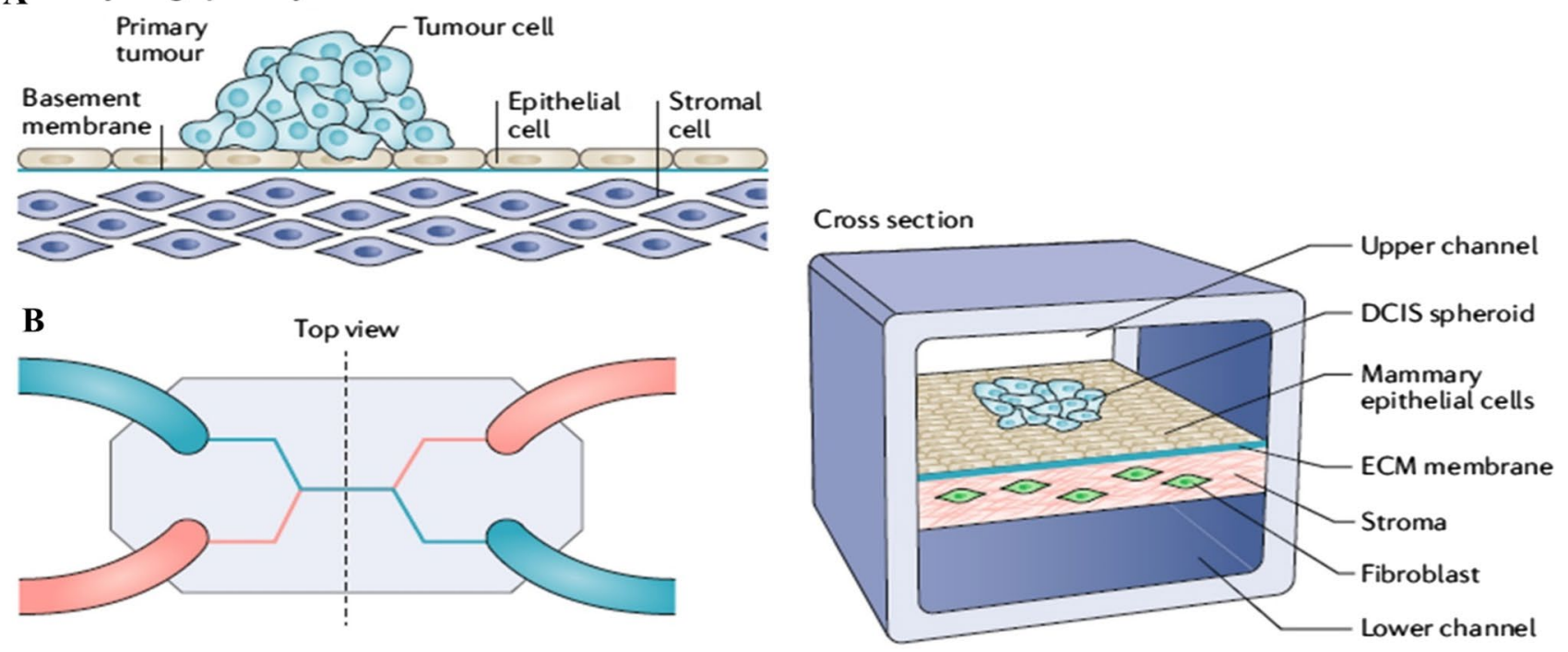

C

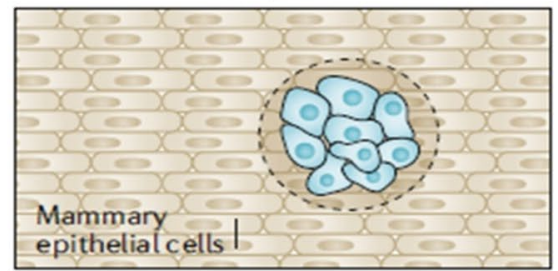

Day 3 control

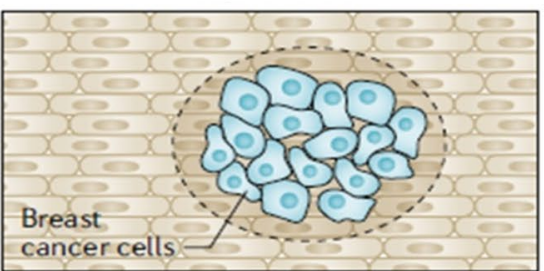

Day 3 paclitaxel

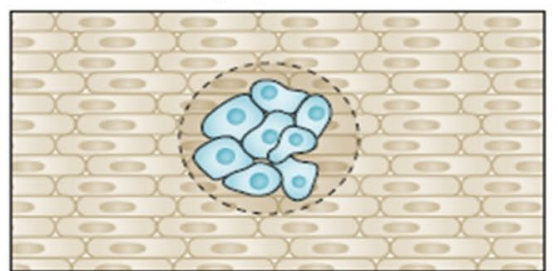

Fig. 13 Orthotopic breast cancer in situ chip. A Early-stage primary tumor formation within a normal epithelium with no breach of the basement membrane. B Top (left) and cross-sectional (right) views of microfluidic organ-on-chip (organ chip) device to model the microarchitecture of ductal carcinoma in situ (DCIS) of the breast in vitro. C Schematic representation of fluorescently labeled DCIS spheroids

when cultured alone or with HMFs under different ECM conditions.

These microfluidic devices have the ability to model interactions between breast cancer cells and relevant stromal microenvironments of both primary and metastatic breast cancer sites. Based on this property, recently a microfluidic model has been designed that utilizes a heterotypic co-culture approach including up to three different cell types (breast cancer cells, stromal cells and monocytes) in combination with gene expression analysis to understand the mechanism of interaction of different cell types through paracrine signaling via production of transforming growth factor- $\beta$ (TGF $\beta$ ) by breast cancer cells and expression of corresponding receptors by stromal cells (Regier et al. 2016).

The microfluidic chips are also reported to investigate with high resolution of the complex interactions between multiple cancer-associated cell types and ECM molecules that are found in the local tissue microenvironment. For example, Hassell et al. developed a human lung cancer chip that recapitulated tumor growth, invasion patterns showing proliferation of breast cancer cells from day 0 to day 3 under control and paclitaxel chemotherapy-treated conditions are shown. The study showed that paclitaxel prevents growth of DCIS spheroids without producing toxic effects on the normal mammary epithelium (Sontheimer-Phelps et al. 2019). Copyright @ copyright 2019, nature reviews cancer

and responses to therapy observed in patients. The chip was based on principle that the rapid growth of human non-small cell lung cancer (NSCLC) cells were entirely dependent on local microenvironmental factors produced by normal lung alveolar epithelial cells and lung endothelial cells that were interfaced across a porous ECM-coated membrane and cultured under an air-liquid interface within a lung alveolus chip (Fig. 15). In this lung cancer chip, conditioned medium from the healthy alveolar epithelium was reported as efficient medium to promote cancer cell proliferation even though secreted factors from the endothelial cells partially suppressed tumor growth. Interestingly, when cyclic strain was exerted on the alveolar-capillary interface of the lung alveolus chip by applying cyclic suction to hollow side chambers of the flexible device to mimic physiological breathing motions, cancer growth was inhibited by $50 \%$. This was found to be mediated by mechanical strain-induced changes in epithelial growth factor receptor (EGFR) phosphorylation, which 

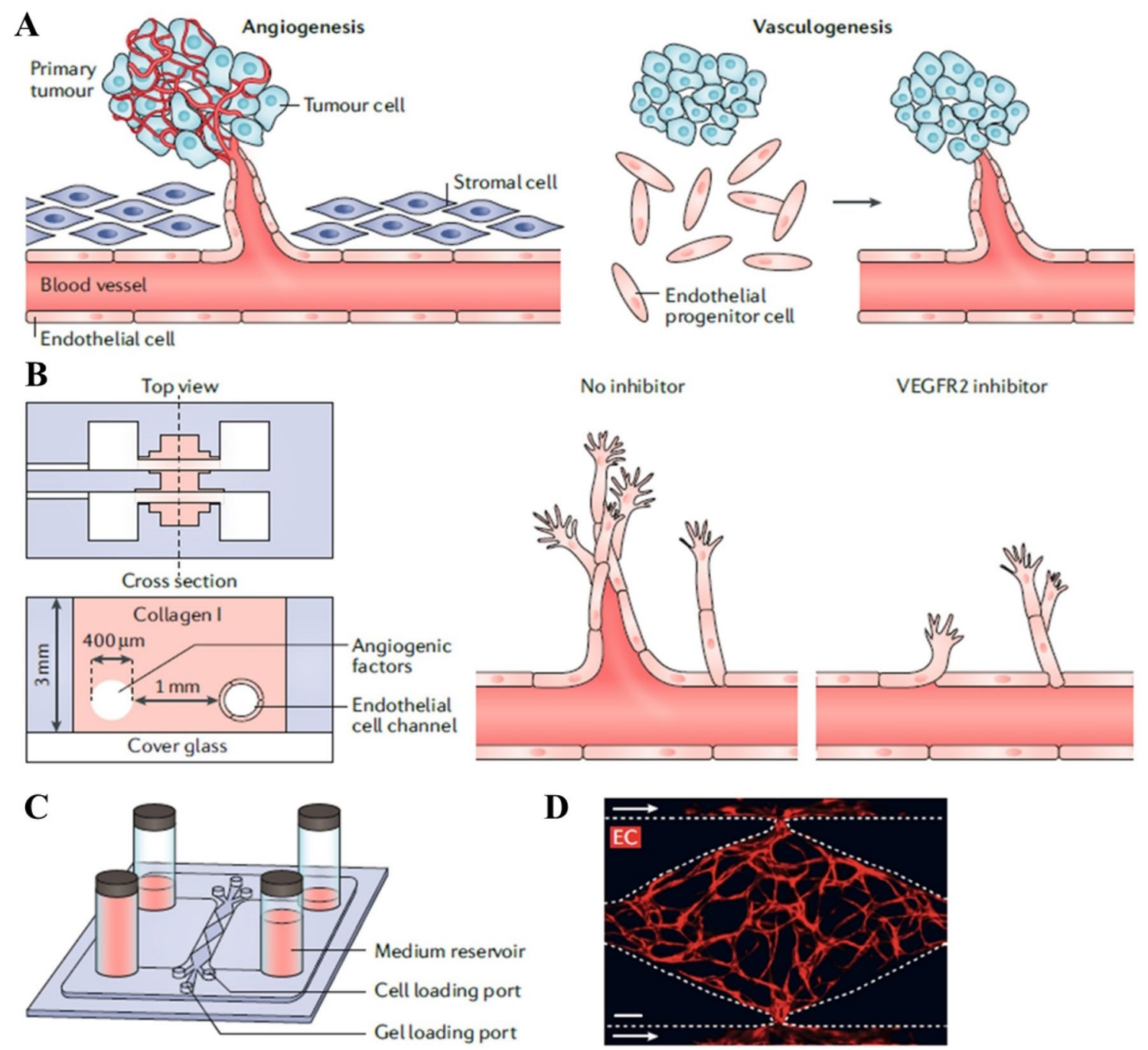

Fig. 14 Neovascularization chips. A The figure shows about angiogenesis and vasculogenesis. B A schematic representation of a microfluidic device that enables analysis of sprouting capillary and new microvessel formation during angiogenesis in vitro is shown (left) (Nguyen et al. 2013). C A schematic of a microfluidic device for generating and studying self-organized microvascular networks dur-

also resulted in increased resistance to the third-generation tyrosine kinase inhibitor rociletinib (Hassell et al. 2017).

Lanz et al., reported a malignant growth treatment of a breast tissue directed on a 3D high-throughput perfused microfluidic stage. Among the different disease conditions, breast malignancy is the most widely recognized intrusive malignancy in women. There are a couple of models that are usually utilized in the treatment. However, restorative reactions were neglected. In this particular trial, they have presented a microfluidic organoplate stage as the choice of treatment where the extracellular matrix was embedded with tumor culture under perfusion. They utilized triplenegative breast malignancy cell lines. In addition, they had also assessed the cultivating densities, ECM composition,

ing vasculogenesis in vitro is shown (left) (Wang et al. 2016). D Cell migration study along the laminin-coated microfluidic channel and spontaneously form a capillary network inside the ECM gel within the diamond-shaped chamber (Sontheimer-Phelps et al. 2019). Copyright @ copyright 2019, nature reviews cancer

and biomechanical (perfusion/static) conditions. After that, the cells were exposed to anticancer medicaments (paclitaxel, olaparib, and cisplatin) and they found that 3D highthroughput culture showed a better response than the 2D culture technique (Lanz et al. 2017).

Microfluidic chips have also been reported to study neovascularization (angiogenesis) among cancer cells. For instance, Nguyen et al., developed a multiple parallel endothelium-lined microvessels within microfluidic channels by depositing sacrificial materials using a form of 3D printing during gel formation. The endothelial cells were plated on the internal walls of these channels and perfused with culture medium to form the microvessels. When tumor angiogenic factors were perfused through one set of these 

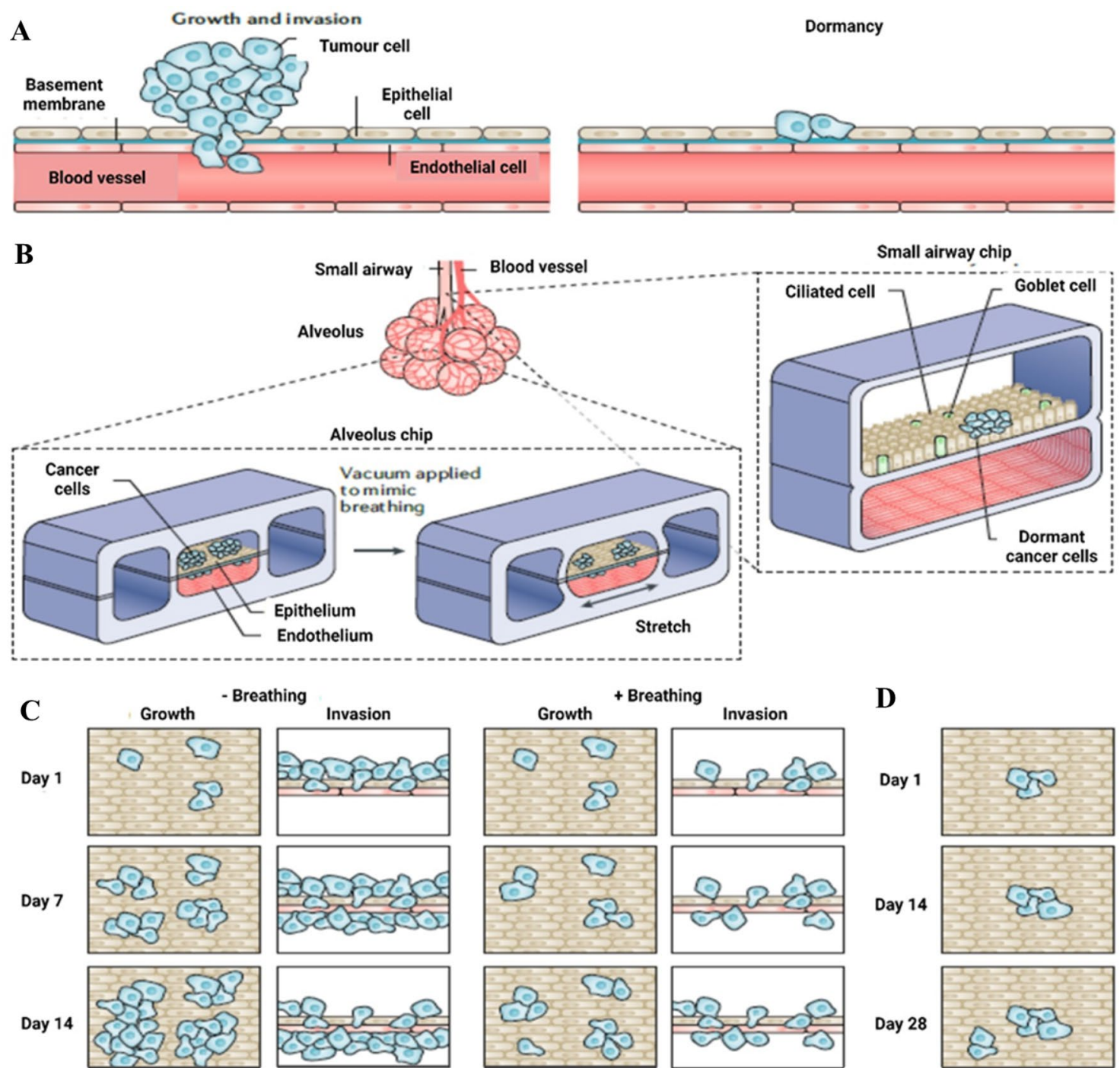

Fig. 15 Orthotopic invasive versus dormant lung cancer organs-onchips. A Proliferation of cancer cells and invasion of the epithelium, underlying basement membrane and endothelium or remain dormant, often depending on the tissue location of the cell of origin (Yousem and Beasley 2007). B Lung alveolus chip (left) and the lung small airway chip (right) used to create two human orthotopic cancer models (Hassell et al. 2017). C Mechanical breathing motions in the lung alveolus chip inhibit both cancer cell growth and invasion through the pores of the ECM-coated membrane and into the vascular channel below (Hassell et al. 2017). D A 28-day study revealed that the cancer cell mass did not substantially expand in size when viewed at 1,14 and 28 days after addition to the lung small airway chip (SontheimerPhelps et al. 2019). Copyright @ copyright 2019, nature reviews cancer

tumor cell-endothelial cell interactions for investigating glioblastoma (Hsu et al. 2013).

Microfluidic cancer organ chip models have also been used to study steps during cancer progression that are accompanied by an EMT. These were developed by coculturing multiple human pancreatic cancer cell lines (Lee et al. 2018) and human HT-29 colon cancer cells (Jeong et al. 2016) with their corresponding cancer-specific fibroblasts. A microfluidic device was designed with multiple parallel channels and the tumor spheroids were loaded in the central channel. The medium was flowed through the surrounding channels and fibroblasts were cultured in the 


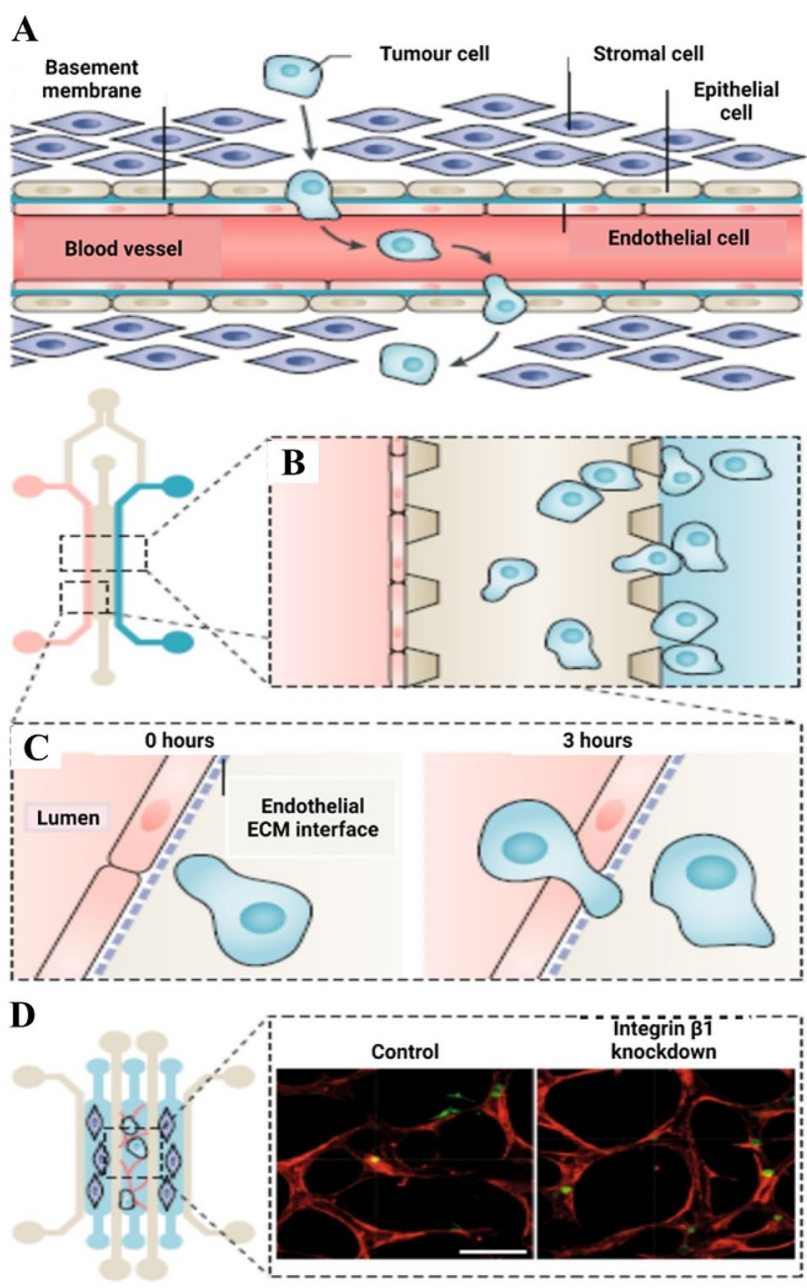

Fig. 16 Metastatic cancer cell dissemination models. A Dissemination of metastatic cancer cells by invading tissue boundaries, intravasating into blood vessels, circulating through the vasculature and then extravasating at a distant site to form a new metastatic lesion. B Cross-section image of the three channels of the device, showing cancer cell dissemination towards the channel lined with endothelial cells. C Schematic representation of the device depicting the interface between the endothelial and extracellular matrix (ECM) gel channels. D Image of a microfluidic microvascular network platform that enables analysis of cancer cell (human MDA-MB-231 breast cancer cells) extravasation (Sontheimer-Phelps et al. 2019). Copyright 2019, nature reviews cancer

outer channels. This setup allowed close proximity coculture without direct cell contact to investigate the role of soluble factors. Induction of EMT occurred only when the fibroblasts were cultured in close proximity to the cancer cells (Jeong et al. 2016; Lee et al. 2018). The induction of this mesenchymal phenotype diminished the response of the cancer cells to chemotherapy upon treatment using paclitaxel (Jeong et al. 2016).

The use of microfluidic culture technologies has also been used to study tumor cell migration and invasiveness. For example, a microfluidic chip equipped with pneumatic micro-valves was used to analyze the paracrine loop between human CL1-0 lung adenocarcinoma cells and MRC-5 lung fibroblasts. Similarly, the primary human macrophages were co-cultured with human MDA-MB-231 breast or PC3 prostate tumor cells or MDA-MB-435S melanoma cells in a microfluidic device to understand cancer cell migration speed and persistence ( $\mathrm{Li}$ et al. 2017).

Tumor cell invasion was studied on-chip by measuring invadopodia formation and ECM degradation by human lung cancer A549 cells growing within an ECM gel-filled microfluidic channel (Wang et al. 2013). In addition, the microfluidic device has been used to evaluate the metastasis of breast cancer cells using metastatic human MDA-MB-231 breast cancer cells and mouse RAW 264.1 macrophages. These cells were placed within neighboring ECM gels which were composed of collagen type I and Matrigel, respectively, within a microfluidic device. The invasion of macrophages was reported into the gels in the presence of breast cancer cells. Whereas, no invasion of macrophages was observed in the absence of breast cancer cells (Huang et al. 2009).

Recently, microfluidic paper-based analytical devices ( $\mu$ PADs) have got popularity as potential alternative tools for various analytical tasks due to certain advantages such as use of paper as the substrate material, which is economical, disposable, easy to fabricate and possibility of external powerfree sample transport driven by capillary forces (Xia et al. 2016). Owing to these advantages, Tenda et al., designed $\mu$ PADs for the colorimetric analysis of sub-microliter sample volumes of human serum albumin (HSA). In this study, calibration curves for HAS were recorded from submicroliter samples $(0.8 \mu \mathrm{L})$, with tolerance against $\pm 0.1 \mu \mathrm{L}$ variations in the applied liquid volume (Tenda et al. 2016). The obtained results showed that the $\mu$ PADs are specific and sensitive for trace volume (sub-micron) of sample.

\subsection{Microfluidic technology used for diagnostic purpose}

Liu et al., worked on establishing a novel electrochemical identification stage by microscopically integrating with a microfluidic chip. They used this novel platform for tracing three therapeutic drugs: warfarin sodium (WFS), cyclophosphamide, and carbamazepine. Warfarin sodium is an oral anticoagulant drug, whereas cyclophosphamide and carbamazepine are used as an immunosuppressant and as an anticonvulsant, respectively. Two methods, namely electrochemical catalysis and gate effect were used for the measurement of WFS. In light of the molecular imprinted technique, the novel electrochemical detection stage was installed with a counter and a reference electrode which employed a platinum wire. For the working anode, nanoporous $\mathrm{Au}-\mathrm{Ag}$ alloy microwire joined with molecularly imprinted polymer was employed. The electrochemical catalysis method is a direct 
detection method where the electrode is used. The sample mixture which had the WFS was siphoned into the microfluidic chip and estimated by differential pulse voltammetry. On the other hand, the gate effect is an indirect method that works based on the peak current change towards the probe ions before and after adsorbing WFS. The outcome of the study was that they successfully developed the detection platform for all the three drugs (Liu et al. 2017a, b).

Fan et al., studied the microfluidic digital PCR strategy which enabled a fast pre-birth conclusion of fetal aneuploidy. Aneuploidy is an unstable genomic condition where the chromosome numbers become abnormal. It might be caused because of the solid relationship with fetal premature delivery and stillbirth. For the evaluation of fetal karyotype, traditional cytogenesis is utilized where the fetal cells were collected from amniotic fluid or chorionic villi which are then cultured. Although it provides accurate results, it requires approximately 1-2 weeks. Moreover, with conventional real-time PCR, one threshold cycle corresponds to a twofold change in copy number, which makes it more challenging to measure the smaller changes. This might be the reason for microfluidic digital PCR to be utilized for the quantification of the number of nucleic acids. They utilized 24 amniocentesis and 16 chorionic villus tests for microfluidic digital PCR. For each target chromosome, 360 PCR responses were performed, and further, the quantity of single-molecule amplification was compared with a reference. The outcome indicated that digital PCR precisely distinguished all the instances of fetal trisomy (Fan et al. 2009).

Basiri et al., studied RNA virus detection using microfluidic devices. The technology was an ideal platform to test its applicability in the detection of coronavirus $(\mathrm{CoV})$. The virus is reported to cause severe acute respiratory syndrome. As of now, quantitative reverse transcription (qRT-PCR) is favored for the detection of the corona virus. However, the disadvantages related to qRT-PCR are: it is expensive, time consuming, and insensitive. To control the pandemic scenario, there needs to be an alternate way that can show rapid detection, ease of use, at the same time being affordable. Microfluidic chip-based innovation may be employed which would offer the stage to apply for numerous demonstrative tests such as RT-PCR, nested-PCR, nucleic acid hybridization, enzyme-linked immunosorbent assay (ELISA), fluorescence-based measures, and many more. Microfluidics chips ensure faster reactions (approximately 25-60 $\mathrm{min}$ ) resulting in accurate and precise outcomes. Moreover, it makes the analysis modest and simple to deal with higher affectability. Thus, microfluidic devices end up being a superior elective technique for the detection of RNA infections. Previously, these devices have been used for the detection of many RNA viruses such as subtype of influenza virus $A(H 1 N 1)$, Zika, hepatitis A virus (HAV), human immunodeficiency virus (HIV), and norovirus (Basiri et al. 2020).
Moarefian et al., demonstrated iontophoretic drug conveyance in a microfluidic device. Iontophoresis is a process that deals with drug conveyance by electrophoresis and electroosmosis. It can likewise build the penetrability of the skin with the assistance of an electric flow. It uses low-power electrical voltage and a continuous consistent flow to coordinate a charged medication to the tissue. As of date, the iontophoresis strategy is utilized as a novel methodology in the therapy of malignancy (in vivo). It is ideal to show the low-power electrical fields in all culture frameworks for the streamlining of iontophoretic drug conveyance to the malignancy cells. They planned an iontophoresis-on-chip stage to evaluate carboplatin drug conveyance and to compare the anticancer viability under various voltages and flows. The authors had further utilized an in vitro heparinbased hydrogel microfluidic device to display the development of a charged medication across extracellular matrix and in breast malignancy cell lines. The concentration of the anticancer medication, for example, carboplatin in the tumor extracellular matrix was determined utilizing a small amount of sample. The study concluded that the medication conveyance and the tumor cell demise were expanded when $50 \mathrm{mV}$ DC electrical field and $3 \mathrm{~mA}$ electrical flow were added (Moarefian et al. 2020).

Maria et al., used cross-connected hyaluronic acid nanoparticles (cHANPs) by utilizing a microfluidic stage integrated with magnetic resonance imaging (MRI). As of date, nanostructures are utilized to capture MRI-contrast agents, without the need to synthetically alter the clinically affirmed segments. In this study, the authors developed a microfluidic stage that was used to incorporate cHANPs in which a clinically pertinent gadolinium diethylenetriamine pentaacidic corrosive (Gd-DTPA) was captured. The microfluidic cycle empowered the creation of monodisperse particles of around $35 \mathrm{~nm}$. Fine-tuning of the process parameters were performed to achieve the impedance of Gd-DTPA, which influenced the utilization of hydrophilic-lipophilic balance of surfactants and $\mathrm{pH}$ conditions during polymer precipitation. Microfluidic approaches have several favorable outcomes where it can improve the relaxometric properties of Gd-DTPA stacked in cHANPs with any synthetic alteration. It was discovered that Gd-DTPA stacked cHANPs supported the unwinding rate and this system could be utilized for both, analytical and restorative applications (Russo et al. 2016).

Herr et al., employed microfluidic immunoassays for the quick determination of components in human saliva. They had also adopted the clinical point-of-care (POC) indicative, which allowed fast quantitation of an oral infection biomarker in the human saliva. In POC diagnostic, usually a monolithic disposable cartridge is employed which is intended to function in a conservative analytical instrument. Microfluidic innovation technology was employed which encouraged investigation of hands-free salivation 
by consolidating test pretreatment (filtering, advancement, blending) with electrophoretic immunoassays. This method empowered the quick estimation of analyte concentration in an insignificant quantity of saliva. Utilizing this strategy, they estimated collagen-dividing enzyme matrix metalloproteinase-8 (MMP-8) in salivation from a healthy as well as a periodontally infected individual. Microfluidics incorporated with POC indicative is equipped for rapid finding of proteinaceous sickness biomarkers in biological liquids (Herr et al. 2007).

Liu et al., studied cancer diagnostics by single-exosome counting immunoassays. Exosomes are usually used as a biomarker in cancer diagnostics. The tumor cells shed the exosomes which indicate the presence of cancer. An immunosorbent assay was designed and combined with droplet microfluidics which enabled the digital diagnostics of exosomes. The exosomes were immobilized on magnetic microbeads with the assistance of a sandwich ELISA. The constructed beads were then isolated and encapsulated into a droplet so that each bead should get encapsulated in a droplet. The droplet-based single-exosome counting ELISA model enabled the counting of specific cancer exosomes in cancer diagnostic purposes. In addition, a limit of detection down to 10 enzymes labeled exosomes complex per microliter was achieved. This approach ensured an early diagnosis of cancer (Liu et al. 2018).

Ziober et al., studied a lab-on-chip system for the screening and diagnosis of oral cancer. Oral squamous cell carcinoma (OSCC) is a deadly cancer which is usually diagnosed only at an advanced stage. Around $40 \%$ of all oral cancer cases represents malignant growth in the head and neck region. Furthermore, they also contain squamous cell carcinoma of the oral cavity, for example, the tongue, mouth floor, buccal mucosa, gums and lips. There is no precise, moderate, and reproducible methodology that can recognize the OSCC at an earlier phase. A rapid oral cancer test is needed for mass screening. In addition, the analysis of OSCC requires present-day lab facilities, refined sorts of equipment, and skilled staff. Ziober et al., employed a lab-on-chip device for biomarker-based identification of oral malignancy using a 'spit test'. Magnetic bead cell arrangement and multiplex identification of mRNAs utilizing bio-scanner tags were utilized for this purpose. The spit test showed the presence of ordinary cells along with cancer cells, and lymphocytes. The lymphocytes were then encapsulated into magnetic beads with anti-CD-45. After incubation, the lymphocytes were captured by the magnet. Furthermore, incubation was carried out after the addition of antibody-coated magnetic beads and quantum dots (Q-dots). The cancer cell-based Q-dot complexes were isolated from the solution, with the help of an external magnetic field. Expulsion of unbound Q-dots was accomplished by washing and malignant growth cell limited Q-dots were assessed. Magnetic beads with captured mRNAs were segregated using an external magnetic field and beads with DNA bio-barcodes were added. During incubation, complexes of barcode-magnetic bead sandwiches were formed and captured by a magnetic field. Eventually, the barcode-DNAs were dehybridized and detected by fluorescence using a fiber-optic array (Ziober et al. 2008).

Xie et al., employed a novel methodology where they combined electrochemical microfluidic chips with different biomarkers for the early detection of malignant growth in the stomach. Presently, gastric cancers are the second most reported malignancy in the world. It is difficult to detect the cancer in the earlier phases. To improve the therapeutic outcomes and viability, a multidisciplinary treatment approach is to be employed. It is critical to monitor the condition from an early phase, which may significantly improve the therapeutic outcomes. A disposable electrochemical microfluidic chip joined with numerous biomarkers was constructed by Xie and co-workers. Six types of biomarkers were employed in the detection of gastric malignant growth. These were carcinoembryonic $\mathrm{Ag}$ (CEA), carbohydrate Ag 19-9(CA 19-9), Helicobacter pylori CagA protein (H.P.), P53 oncoprotein (P53), and pepsinogen I and II (PG-I and II). Three specially designed gold electrodes were used to avoid cross-contamination in each of the detection areas. The device consisted of 6 different kinds of biomarkers targeting 6 detection areas. Differential pulse voltammetry (DPV) was employed to compare the electron transfer ability between working electrodes and bare electrodes after antibody immobilization. The biomarker solutions were injected into the microchannel and were then incubated at $37^{\circ} \mathrm{C}$ for $30 \mathrm{~min}$. This methodology has proven to possess an extraordinary potential in early detection of gastric malignancy (Xie et al. 2015).

Fu et al., studied and analyzed a swine virus sample using an incorporated microfluidic stage. The microfluidic stage incorporated a microfluidic chip combined with a semi-automated immunoassay system. Currently, the ELISA technique is the most commonly employed technique for the detection of swine virus. Samples such as a serum or oral fluid are usually collected and tested through an ELISA. However, the main disadvantage with this technique is that it is tedious and time consuming. To confirm the presence of the virus, a rapid, inexpensive, and reproducible technique is needed. Microfluidic chips based platforms provide accurate and early detection of the viral particles. These are rapid and have a high analytical effectiveness. A single chip could be employed for the analysis of several samples. These chips were used to detect several types of viruses from porcine origin, respiratory syndrome virus, CSF virus, and porcine circovirus type 2 . The procedure includes sample and reagent injection, well washing, waste collection, and finally colorimetric detection. The findings revealed that the microfluidic technique took just $12 \mathrm{~min}$ and $4 \mu \mathrm{L}$ of sample for 
the detection process. The accuracy rate was high with high effectiveness and high explicitness (Fu et al. 2020).

In recent years, numerical approaches have been designed for developing efficient microfluidic technologies for cell analysis (Sheidaei et al. 2020). The numerical based evaluation are powerful tools to rapidly analyze the problems at a lower cost. In addition, these are able to study multiphysics problems that help in understanding biological processes that involve physical phenomena such as fluid flow, solid mechanics, mass and heat transfer. The numerical simulation can assist researchers in studying complex device functions. Moreover, numerical simulations are capable of determining critical operating parameters such as velocity, pressure, concentration, shear rate, and temperature that are cumbersome to be measured experimentally. Looking at various advantages of microfluidic chips, Zahorodny-Burke et al., simulated the stationary convection diffusion mass transfer phenomena in a 2D cell culture device to explore the effect of chip thickness and channel height on oxygen transport (Zahorodny-Burke et al. 2011). A decrease in the oxygen concentration in the cell layer was located at the bottom of the culture channel was noted with increase in heights of the channel and the chip (Fig. 17A). Huang and Nguyen used a 2D computational model to optimize key dimensional parameters of a cyclic cell-stretching device (Huang and Nguyen 2013). The cells were cultured on a thin membrane and a mechanical strain was induced to these cells. The effect of geometrical parameters on membrane displacement was studied (Fig. 17B). Liu et al. (2017c) numerically simulated a 2D gel injection process in a microfluidic chip with different pillar spaces (Fig. 17C). In another numerical study, Chen et al. (2019) investigated a 2D fluid flow in a microfluidic device with various micropillar designs. Pillars with circular, elliptical and square cross-section were arranged in both aligned and staggered styles. It was observed that in the staggered arrangement, the fluid flew through the center of the array as well as around the pillars. For distribution of fluids, the staggered arrangement was found better than the aligned pillars (Fig. 17D)

\section{Conclusion}

The microfluidic chip technology employs advanced innovative strategies and is an emerging science in the field of life sciences and biomedical sciences. It is predominantly utilized for the estimation of behavioral changes in miniature fluids (micro- or nano-level) through miniature channels that are engraved on the polymer layer. The technology enables effective control over the physicochemical responses of the liquid. Due to its miniature scaled down methodology, it brings about high mass exchange and high throughput. It is primarily employed in drug discovery, proteomics, drug screening, clinical analysis and food innovation. Various types of microfluidic chips are currently employed for various purposes. Compared to the conventional methods, microfluidic chip technology offers much advantage and better outcomes in terms of time consumed and amount of samples and reagents required. In pharmaceutical investigation, microfluidic innovation could be integrated with various other detection equipment, namely PCR, ESI-MS, MALDI-MS and GC-MS to name a few. Much of the current applications of microfluidic innovation are reported in the detection and treatment of malignancies. Organ-on-chips are reportedly utilized for the investigation of the physiological processes in specific body organs such as brain, lungs, heart, kidney, gut and skin. Significantly, microfluidic innovation is playing a major role in the current COVID19 pandemic situation, especially in the treatment strategies and analysis of coronavirus particles by integrating with a qRT-PCR strategy. Thus, microfluidic innovation technology has demonstrated that it is a superior cutting-edge technology which could be adopted in the screening and treatment for various infective conditions. Despite enormous advantages, microfluidic chips suffer from some limitations. For example, paper-based microfluidic devices are very complex to pattern the channels on the chip. The organ-on-a-chip systems have replaced animal testing and improved patient safety and considered as a great advancement in medical research because they facilitate testing of active ingredients on cell cultures in the chambers of a plastic chip. However, they are not a true-to-life replication of the human body and can only simulate a few functions and activities because the human body is a complex system where different kinds of organs and cells interact. Its complexity cannot be built into organ-on-a-chip systems. Moreover, their designs are complex. When coupling organs on chips, whether it is to measure the efficiency of a treatment or to study the interactions between organs, the relative size of the models matters. In case of organ-on-chip, sometimes it becomes difficult to determine right scale (organ mass/organ volume/ fluid flow) for analysis. Determination of quantity of fluid inside the organ-on-chip is another limitation. It has been reported that human body contains $5 \mathrm{~L}$ blood, hence, in can be imagined that a microhuman would have $5 \mu \mathrm{L}$ of blood. This total volume of $5 \mu \mathrm{L}$ implies many challenges because excessive volume could dilute the treatment, or hormones secreted by the different organs, and skew the results of the tests. Hence, it is important to create microfluidic pumps and valves of the right size for such small volumes (Wikswo et al. 2013). Hence, there is a need for standardization of microfluidic chips to compare and interpret their findings. Since this is a process starts with the cells that are being used, some researchers use primary cell cultures and other cell lines, while others try to differentiate stem cells into specialized cells for specific organs. Other issues include the 

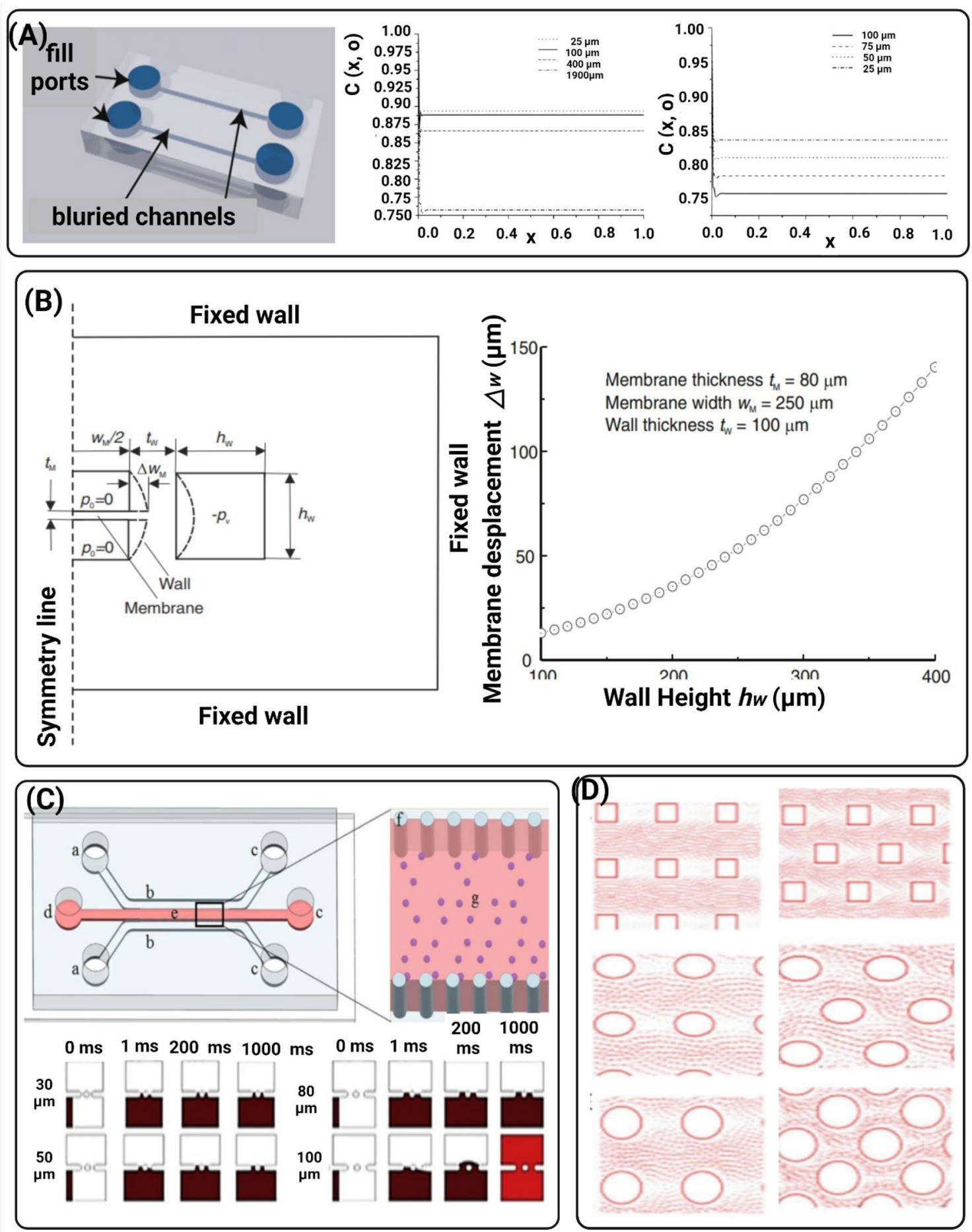

Fig. 17 A (i) Microfluidic cell culture device made of oxygen-permeable polymer. The effect of polymer thickness (ii) and channel height (iii) on the oxygen concentration in the cell layer (Zahorodny-Burke et al. 2011) Copyright 2017, Elsevier. B (i) The cross-section of the microfluidic device and the investigated geometrical parameters. (ii) The effect of wall height on the horizontal displacement of the membrane arrangement (Huang and Nguyen 2013). C (i) A microfluidic chip with a middle gel and two lateral media channels. The gel channel is separated from the media channels by micropillars. (ii) The effect of pillar space on the process of gel filling within the microfluidic device (numerical simulation) (Jun-Shan et al, 2017) copyright 2017, Elsevier. D Fluid flow through the micropillars with different shapes and arrangements. Cases (i) are for the aligned arrangement and cases (ii) refer to the staggered (Chen et al. 2019) 
formulation of cell culture media, the cell life cycle on the chips or the materials used in bioprinting for example. These are all variables that could impact results. Based on these facts, it can be concluded that microfluidic chips still have a long way to go before they can replicate the complexity of living organisms.

Acknowledgements I would like to express my deep gratitude towards Chancellor of LPU, Shree Ashok Mittal for providing necessary support.

Author contributions PP: methodology, data curation, and writingoriginal draft. SKS: conceptualization, validation, supervision, and writing - review and editing. MG: supervision and writing-review and editing. SV: methodology. BK: methodology. DKC: data curation and writing - original draft. KA: methodology. GG: methodology. NKJ: methodology. PKG: methodology. PP: methodology. KD: methodology. HD: methodology. DK: methodology. VK: methodology.

Funding This research did not receive any specific grant from funding agencies in the public, commercial, or not-for-profit sectors.

Availability of data and materials Not applicable.

\section{Declarations}

Conflict of interest The authors declare that they have no conflict of interests.

Ethics approval Not applicable.

Consent to participate Not applicable.

Consent for publication Yes.

\section{References}

Adams RP (2007) Identification of essential oil components by gas chromatography/mass spectrometry, vol 456. Allured Publishing Corporation, Carol Stream

Ahrberg CD, Manz A, Chung BG (2016) Polymerase chain reaction in microfluidic devices. Lab Chip 16(20):3866-3884

Akshatha HS, Gurupadayya BM (2018) Application of liquid chromatography coupled with mass spectrometry in the impurity profiling of drug substances and products. Asian J Pharm Clin Res 11(5):30-37

Andersson H, Van Den Berg A (2004) Microfabrication and microfluidics for tissue engineering: state of the art and future opportunities. Lab Chip 4(2):98-103

Annabi N, Selimović Š, Cox JPA, Ribas J, Bakooshli MA, Heintze D, Weiss AS, Cropek D, Khademhosseini A (2013) Hydrogelcoated microfluidic channels for cardiomyocyte culture. Lab Chip 13(18):3569-3577

Ashammakhi N, Nasiri R, De Barros NR, Tebon P, Thakor J, Goudie M, Shamloo A, Martin MG, Khademhosseni A (2020) Gut-ona-chip: current progress and future opportunities. Biomaterials. https://doi.org/10.1016/j.biomaterials.2020.120196

Bakooshli MA, Heintze D, Weiss AS, Cropekk D, Khademhosseini A (2013) Hydrogel-coated microfluidic channels for cardiomyocyte culture3. Lab Chip 13:3569-3577
Bang S, Jeong S, Choi N, Kim HN (2019) Brain-on-a-chip: a history of development and future perspective. Biomicrofluidics 13(5):051301. https://doi.org/10.1063/1.5120555

Baret J-C (2012) Surfactants in droplet-based microfluidics. Lab Chip 12(3):422-433

Basiri A, Heidari A, Nadi MF, Fallahy MTP, Nezamabadi SS, Sedighi M, Saghazadeh A, Rezaei N (2020) Microfluidic devices for detection of RNA viruses. Rev Med Virol. https://doi.org/10. 1002/rmv.2154

Beebe DJ, Moore JS, Bauer JM, Yu Q, Liu RH, Devadoss C, Jo B-H (2000) Functional hydrogel structures for autonomous flow control inside microfluidic channels. Nature 404(6778):588-590

Berthier J, Brakke KA, Berthier E (2016) Open microfluidics. Wiley, Hoboken

Bruzewicz DA, McGuigan AP, Whitesides GM (2008) Fabrication of a modular tissue construct in a microfluidic chip. Lab Chip 8(5):663-671

Carvalho MR, Lima D, Reis RL, Correlo VM, Oliveira JM (2015) Evaluating biomaterial-and microfluidic-based 3D tumor models. Trends Biotechnol 33(11):667-678

Chatterjee D, Ytterberg AJ, Son SU, Loo JA, Garrell RL (2010) Integration of protein processing steps on a droplet microfluidics platform for MALDI-MS analysis. Anal Chem 82(5):2095-2101

Chen Q, Wu J, Zhang Y, Lin J-M (2012) Qualitative and quantitative analysis of tumor cell metabolism via stable isotope labeling assisted microfluidic chip electrospray ionization mass spectrometry. Anal Chem 84(3):1695-1701

Chen WX, Li JG, Wan XH, Zou XS, Qi SY, Zhang YQ, Weng QM, Li JY, Xiong WM, Xie C (2019) Design of a microfluidic chip consisting of micropillars and its use for the enrichment of nasopharyngeal cancer cells. Oncol Lett 17(2):1581-1588

Chen C, Gu Y, Philippe J, Zhang P, Bachman H, Zhang J, Mai J, Rufo J, Rawls JF, Davis EE (2021) Acoustofluidic rotational tweezing enables high-speed contactless morphological phenotyping of zebrafish larvae. Nat Commun 12(1):1-13

Cheong R, Paliwal S, Levchenko A (2010) High-content screening in microfluidic devices. Expert Opin Drug Discov 5(8):715-720

Choi K, Ng AH, Fobel R, Wheeler AR (2012) Digital microfluidics. Annu Rev Anal Chem 5:413-440

Choudhury D, van Noort D, Iliescu C, Zheng B, Poon K-L, Korzh S, Korzh V, Yu H (2012) Fish and chips: a microfluidic perfusion platform for monitoring zebrafish development. Lab Chip 12(5):892-900

Chronis N, Zimmer M, Bargmann CI (2007) Microfluidics for in vivo imaging of neuronal and behavioral activity in Caenorhabditis elegans. Nat Methods 4(9):727-731

Chung C, Degner B, Decker EA, McClements DJ (2013) Oil-filled hydrogel particles for reduced-fat food applications: fabrication, characterization, and properties. Innov Food Sci Emerg Technol 20:324-334

Crane MM, Chung K, Stirman J, Lu H (2010) Microfluidics-enabled phenotyping, imaging, and screening of multicellular organisms. Lab Chip 10(12):1509-1517

Cuchiara MP, Allen AC, Chen TM, Miller JS, West JL (2010) Multilayer microfluidic PEGDA hydrogels. Biomaterials 31(21):5491-5497

Cui P, Wang S (2019) Application of microfluidic chip technology in pharmaceutical analysis: a review. J Pharm Anal 9(4):238-247

Damiati S, Kompella UB, Damiati SA, Kodzius R (2018) Microfluidic devices for drug delivery systems and drug screening. Genes 9(2): 103

Dendukuri D, Pregibon DC, Collins J, Hatton TA, Doyle PS (2006) Continuous-flow lithography for high-throughput microparticle synthesis. Nat Mater 5(5):365-369

DeVoe DL, Lee CS (2006) Microfluidic technologies for MALDI-MS in proteomics. Electrophoresis 27(18):3559-3568 
Dittrich PS, Manz A (2006) Lab-on-a-chip: microfluidics in drug discovery. Nat Rev Drug Discov 5(3):210-218

Fan HC, Blumenfeld YJ, El-Sayed YY, Chueh J, Quake SR (2009) Microfluidic digital PCR enables rapid prenatal diagnosis of fetal aneuploidy. Am J Obstet Gynecol 200(5):543.e541-543. e547

Feng X, Liu BF, Li J, Liu X (2015) Advances in coupling microfluidic chips to mass spectrometry. Mass Spectrom Rev 34(5):535-557

Feng S, Shirani E, Inglis DW (2019) Droplets for sampling and transport of chemical signals in biosensing: a review. Biosensors 9(2):80

Focaroli S, Mazzitelli S, Falconi M, Luca G, Nastruzzi C (2014) Preparation and validation of low cost microfluidic chips using a shrinking approach. Lab Chip 14(20):4007-4016

Franzen N, van Harten WH, Retèl VP, Loskill P, van den Eijndenvan Raaij J, IJzerman M (2019) Impact of organ-on-a-chip technology on pharmaceutical R\&D costs. Drug Discov Today 24(9):1720-1724

Fu T, Wu Y, Ma Y, Li HZ (2012) Droplet formation and breakup dynamics in microfluidic flow-focusing devices: from dripping to jetting. Chem Eng Sci 84:207-217

Fu Y, Li W, Dai B, Zheng L, Zhang Z, Qi D, Cheng X, Zhang D, Zhuang S (2020) Diagnosis of mixed infections with swine viruses using an integrated microfluidic platform. Sens Actuators B Chem 312:128005

Gao D, Liu H, Jiang Y, Lin J-M (2013) Recent advances in microfluidics combined with mass spectrometry: technologies and applications. Lab Chip 13(17):3309-3322

Gao H, Yan C, Wu W, Li J (2020) Application of microfluidic chip technology in food safety sensing. Sensors 20(6): 1792

Garstecki P, Fuerstman MJ, Stone HA, Whitesides GM (2006) Formation of droplets and bubbles in a microfluidic T-junction-scaling and mechanism of break-up. Lab Chip 6(3):437-446

Ge A, Hu L, Wang X, Zhu J, Feng X, Du W, Liu B-F (2018) Logarithmic bacterial gradient chip for analyzing the effects of dietary restriction on C. elegans growth. Sens Actuators B Chem 255:735-744

Grandfils E, Cavallasca J, Casquillas GV (2020) Gut-on-chip: keeping up with the technology

Grinias JP, Kennedy RT (2016) Advances in and prospects of microchip liquid chromatography. TrAC Trends Anal Chem 81:110-117

Grosberg A, Alford PW, McCain ML, Parker KK (2011) Ensembles of engineered cardiac tissues for physiological and pharmacological study: heart on a chip. Lab Chip 11(24):4165-4173

Guo Q, Zhang L, Liu J, Li Z, Li J, Zhou W, Wang H, Li J, Liu D, Yu X (2021) Multifunctional microfluidic chip for cancer diagnosis and treatment. Nanotheranostics 5(1):73

Harrison RG, Greenman M, Mall FP, Jackson C (1907) Observations of the living developing nerve fiber. Anat Rec 1(5):116-128

Hassell BA, Goyal G, Lee E, Sontheimer-Phelps A, Levy O, Chen CS, Ingber DE (2017) Human organ chip models recapitulate orthotopic lung cancer growth, therapeutic responses, and tumor dormancy in vitro. Cell Rep 21(2):508-516

Herr AE, Hatch AV, Throckmorton DJ, Tran HM, Brennan JS, Giannobile WV, Singh AK (2007) Microfluidic immunoassays as rapid saliva-based clinical diagnostics. Proc Natl Acad Sci 104(13):5268-5273

Hodzic E (2016) Single-cell analysis: advances and future perspectives. Bosn J Basic Med Sci 16(4):313

Hsu Y-H, Moya ML, Hughes CC, George SC, Lee AP (2013) A microfluidic platform for generating large-scale nearly identical human microphysiological vascularized tissue arrays. Lab Chip 13(15):2990-2998

Huang Y, Nguyen N-T (2013) A polymeric cell stretching device for real-time imaging with optical microscopy. Biomed Microdevice 15(6):1043-1054
Huang CP, Lu J, Seon H, Lee AP, Flanagan LA, Kim H-Y, Putnam AJ, Jeon NL (2009) Engineering microscale cellular niches for three-dimensional multicellular co-cultures. Lab Chip 9(12):1740-1748

Huft J, Haynes CA, Hansen CL (2013) Microfluidic integration of parallel solid-phase liquid chromatography. Anal Chem 85(5):2999-3005

Huh D (2015) A human breathing lung-on-a-chip. Ann Am Thorac Soc 12(Supplement 1):S42-S44

Hwang H, Lu H (2013) Microfluidic tools for developmental studies of small model organisms-nematodes, fruit flies, and zebrafish. Biotechnol J 8(2):192-205

Ingber DE (2011) The Wyss Institute at Harvard University. IEEE Pulse 2(4):43-46

Jahn A, Reiner JE, Vreeland WN, DeVoe DL, Locascio LE, Gaitan M (2008) Preparation of nanoparticles by continuous-flow microfluidics. J Nanopart Res 10(6):925-934

Jahromi MAM, Abdoli A, Rahmanian M, Bardania H, Bayandori M, Basri SMM, Kalbasi A, Aref AR, Karimi M, Hamblin MR (2019) Microfluidic brain-on-a-chip: perspectives for mimicking neural system disorders. Mol Neurobiol 56(12):8489-8512

Jeong S-Y, Lee J-H, Shin Y, Chung S, Kuh H-J (2016) Co-culture of tumor spheroids and fibroblasts in a collagen matrix-incorporated microfluidic chip mimics reciprocal activation in solid tumor microenvironment. PLoS One 11(7):e0159013. https://doi.org/ 10.1371/journal.pone.0159013

Kaler KV, Prakash R (2014) Droplet microfluidics for chip-based diagnostics. Sensors 14(12):23283-23306

Kang L, Chung BG, Langer R, Khademhosseini A (2008) Microfluidics for drug discovery and development: from target selection to product lifecycle management. Drug Discov Today 13(1-2):1-13

Kang D-K, Ali MM, Zhang K, Pone EJ, Zhao W (2014) Droplet microfluidics for single-molecule and single-cell analysis in cancer research, diagnosis and therapy. TrAC Trends Anal Chem 58:145-153

Killeen K, Yin H, Sobek D, Brennen R, Van de Goor T (2003) ChipLC/MS: HPLC-MS using polymer microfluidics. Proc MicroTAS 9:481-484

Kim S, Takayama S (2015) Organ-on-a-chip and the kidney. Kidney Res Clin Pract 34(3):165-169

Kim HJ, Huh D, Hamilton G, Ingber DE (2012) Human gut-on-a-chip inhabited by microbial flora that experiences intestinal peristalsis-like motions and flow. Lab Chip 12(12):2165-2174

Kimura H, Sakai Y, Fujii T (2018) Organ/body-on-a-chip based on microfluidic technology for drug discovery. Drug Metab Pharmacokinet 33(1):43-48

Koo H-J, Velev OD (2017) Design and characterization of hydrogelbased microfluidic devices with biomimetic solute transport networks. Biomicrofluidics 11(2):024104

Koster S, Verpoorte E (2007) A decade of microfluidic analysis coupled with electrospray mass spectrometry: an overview. Lab Chip 7(11):1394-1412

Kussmann M, Roepstorff P (2000) Sample preparation techniques for peptides and proteins analyzed by MALDI-MS. In: Mass spectrometry of proteins and peptides. Springer, Berlin, pp 405-424

Küster SK, Fagerer SR, Verboket PE, Eyer K, Jefimovs K, Zenobi R, Dittrich PS (2013) Interfacing droplet microfluidics with matrixassisted laser desorption/ionization mass spectrometry: label-free content analysis of single droplets. Anal Chem 85(3):1285-1289

Lanz HL, Saleh A, Kramer B, Cairns J, Ng CP, Yu J, Trietsch SJ, Hankemeier T, Joore J, Vulto P (2017) Therapy response testing of breast cancer in a 3D high-throughput perfused microfluidic platform. BMC Cancer 17(1):1-11

Lazar IM, Trisiripisal P, Sarvaiya HA (2006) Microfluidic liquid chromatography system for proteomic applications and biomarker screening. Anal Chem 78(15):5513-5524 
Leclerc E, Sakai Y, Fujii T (2003) Cell culture in 3-dimensional microfluidic structure of PDMS (polydimethylsiloxane). Biomed Microdevice 5(2):109-114

Lee J, Kim S (2018) Kidney-on-a-chip: a new technology for predicting drug efficacy, interactions, and drug-induced nephrotoxicity. Curr Drug Metab 19(7):577-583

Lee J, Soper SA, Murray KK (2009a) Microfluidic chips for mass spectrometry-based proteomics. J Mass Spectrom 44(5):579-593

Lee W, Debasitis JC, Lee VK, Lee J-H, Fischer K, Edminster K, Park J-K, Yoo S-S (2009b) Multi-layered culture of human skin fibroblasts and keratinocytes through three-dimensional freeform fabrication. Biomaterials 30(8):1587-1595

Lee WG, Kim Y-G, Chung BG, Demirci U, Khademhosseini A (2010) Nano/microfluidics for diagnosis of infectious diseases in developing countries. Adv Drug Deliv Rev 62(4-5):449-457

Lee V, Singh G, Trasatti JP, Bjornsson C, Xu X, Tran TN, Yoo S-S, Dai G, Karande P (2014) Design and fabrication of human skin by three-dimensional bioprinting. Tissue Eng Part C Methods 20(6):473-484

Lee J-H, Kim S-K, Khawar IA, Jeong S-Y, Chung S, Kuh H-J (2018) Microfluidic co-culture of pancreatic tumor spheroids with stellate cells as a novel 3D model for investigation of stromamediated cell motility and drug resistance. J Exp Clin Cancer Res 37(1):1-12

Leonelli S, Ankeny RA (2013) What makes a model organism? Endeavour 37(4):209-212

Li XJ, Zhou Y (2013) Microfluidic devices for biomedical applications. Elsevier, Amsterdam

Li Y, Yang F, Chen Z, Shi L, Zhang B, Pan J, Li X, Sun D, Yang H (2014) Zebrafish on a chip: a novel platform for real-time monitoring of drug-induced developmental toxicity. PLoS ONE 9(4):e94792

Li R, Hebert JD, Lee TA, Xing H, Boussommier-Calleja A, Hynes RO, Lauffenburger DA, Kamm RD (2017) Macrophage-secreted TNF $\alpha$ and TGF $\beta 1$ influence migration speed and persistence of cancer cells in 3D tissue culture via independent pathways. Can Res 77(2):279-290

Lin Y, Gritsenko D, Feng S, Teh YC, Lu X, Xu J (2016) Detection of heavy metal by paper-based microfluidics. Biosens Bioelectron $83: 256-266$

Liu D, Zhang H, Fontana F, Hirvonen JT, Santos HA (2017a) Microfluidic-assisted fabrication of carriers for controlled drug delivery. Lab Chip 17(11):1856-1883

Liu J, Zhang Y, Jiang M, Tian L, Sun S, Zhao N, Zhao F, Li Y (2017b) Electrochemical microfluidic chip based on molecular imprinting technique applied for therapeutic drug monitoring. Biosens Bioelectron 91:714-720

Liu J-S, Zhang Y-Y, Wang Z, Deng J-Y, Ye X, Xue R-Y, Ge D, Xu Z (2017c) Design and validation of a microfluidic chip with micropillar arrays for three-dimensional cell culture. Chin J Anal Chem 45(8):1109-1114

Liu C, Xu X, Li B, Situ B, Pan W, Hu Y, An T, Yao S, Zheng L (2018) Single-exosome-counting immunoassays for cancer diagnostics. Nano Lett 18(7):4226-4232

Maher S, Santos A, Kumeria T, Kaur G, Lambert M, Forward P, Evdokiou A, Losic D (2017) Multifunctional microspherical magnetic and $\mathrm{pH}$ responsive carriers for combination anticancer therapy engineered by droplet-based microfluidics. J Mater Chem B 5(22):4097-4109

Mashaghi S, Abbaspourrad A, Weitz DA, van Oijen AM (2016) Droplet microfluidics: a tool for biology, chemistry and nanotechnology. TrAC Trends Anal Chem 82:118-125

Mathur A, Loskill P, Shao K, Huebsch N, Hong S, Marcus SG, Marks N, Mandegar M, Conklin BR, Lee LP (2015) Human iPSC-based cardiac microphysiological system for drug screening applications. Sci Rep 5:8883
Mathur L, Ballinger M, Utharala R, Merten CA (2020) Microfluidics as an enabling technology for personalized cancer therapy. Small 16(9):1904321. https://doi.org/10.1002/smll.201904321

Matsusaki M, Case CP, Akashi M (2014) Three-dimensional cell culture technique and pathophysiology. Adv Drug Deliv Rev 74:95-103

McDonald JC, Duffy DC, Anderson JR, Chiu DT, Wu H, Schueller OJ, Whitesides GM (2000) Fabrication of microfluidic systems in poly (dimethylsiloxane). Electrophor Int J 21(1):27-40

Moarefian M, Davalos RV, Tafti DK, Achenie LE, Jones CN (2020) Modeling iontophoretic drug delivery in a microfluidic device. Lab Chip 20(18):3310-3321

Montanez-Sauri SI, Sung KE, Berthier E, Beebe DJ (2013) Enabling screening in 3D microenvironments: probing matrix and stromal effects on the morphology and proliferation of T47D breast carcinoma cells. Integr Biol 5(3):631-640

Mouradian S (2002) Lab-on-a-chip: applications in proteomics. Curr Opin Chem Biol 6(1):51-56

Nguyen D-HT, Stapleton SC, Yang MT, Cha SS, Choi CK, Galie PA, Chen CS (2013) Biomimetic model to reconstitute angiogenic sprouting morphogenesis in vitro. Proc Natl Acad Sci 110(17):6712-6717

Nguyen HT, Marquis M, Anton M, Marze S (2019) Studying the real-time interplay between triglyceride digestion and lipophilic micronutrient bioaccessibility using droplet microfluidics. 2 Application to various oils and (pro) vitamins. Food Chem 275:661-667

Nie J, Gao Q, Wang Y, Zeng J, Zhao H, Sun Y, Shen J, Ramezani H, Fu Z, Liu Z (2018) Vessel-on-a-chip with hydrogel-based microfluidics. Small 14(45):1802368. https://doi.org/10.1002/ smll.201802368

Opalski AS, Kaminski TS, Garstecki P (2019) Droplet microfluidics as a tool for the generation of granular matters and functional emulsions. Kona Powder Part J 36:50-71

Ottesen EA, Hong JW, Quake SR, Leadbetter JR (2006) Microfluidic digital PCR enables multigene analysis of individual environmental bacteria. Science 314(5804):1464-1467

Pang X, Lewis AC (2012) A microfluidic lab-on-chip derivatisation technique for the measurement of gas phase formaldehyde. Anal Methods 4(7):2013-2020

Pang X, Lewis AC, Rodenas-Garcia M (2013) Microfluidic lab-on-achip derivatization for gaseous carbonyl analysis. J Chromatogr A 1296:93-103

Pang X, Carpenter LJ, Lewis AC (2015) Microfluidic derivatisation technique for determination of gaseous molecular iodine with GC-MS. Talanta 137:214-219

Park S, Zhang Y, Lin S, Wang T-H, Yang S (2011) Advances in microfluidic PCR for point-of-care infectious disease diagnostics. Biotechnol Adv 29(6):830-839

Pihl J, Karlsson M, Chiu DT (2005) Microfluidic technologies in drug discovery. Drug Discov Today 10(20):1377-1383

Probst C, Schneider S, Loskill P (2018) High-throughput organ-ona-chip systems: current status and remaining challenges. Curr Opin Biomed Eng 6:33-41

Regier MC, Maccoux LJ, Weinberger EM, Regehr KJ, Berry SM, Beebe DJ, Alarid ET (2016) Transitions from mono-to co-to tri-culture uniquely affect gene expression in breast cancer, stromal, and immune compartments. Biomed Microdevice 18(4):1-14

Rivet C, Lee H, Hirsch A, Hamilton S, Lu H (2011) Microfluidics for medical diagnostics and biosensors. Chem Eng Sci 66(7):1490-1507

Rohde CB, Zeng F, Gonzalez-Rubio R, Angel M, Yanik MF (2007) Microfluidic system for on-chip high-throughput whole-animal sorting and screening at subcellular resolution. Proc Natl Acad Sci 104(35):13891-13895 
Roper MG, Shackman JG, Dahlgren GM, Kennedy RT (2003) Microfluidic chip for continuous monitoring of hormone secretion from live cells using an electrophoresis-based immunoassay. Anal Chem 75(18):4711-4717

Rosser J, Calvo I, Schlager M, Purtscher M, Jenner F, Ertl P (2015) Recent advances of biologically inspired 3D microfluidic hydrogel cell culture systems. J Cell Biol Cell Metab 2(5):1-14

Russo M, Bevilacqua P, Netti PA, Torino E (2016) A microfluidic platform to design crosslinked hyaluronic acid nanoparticles (cHANPs) for enhanced MRI. Sci Rep 6(1):1-10

Seemann R, Brinkmann M, Pfohl T, Herminghaus S (2011) Droplet based microfluidics. Rep Prog Phys 75(1):016601. https://doi. org/10.1088/0034-4885/75/1/016601

Sheidaei Z, Akbarzadeh P, Kashaninejad N (2020) Advances in numerical approaches for microfluidic cell analysis platforms. J Sci Adv Mater Devices 5(3):295-307

Shen C, Li Y, Wang Y, Meng Q (2019) Non-swelling hydrogel-based microfluidic chips. Lab Chip 19(23):3962-3973

Shi W, Wen H, Lin B, Qin J (2011) Microfluidic platform for the study of Caenorhabditis elegans. Top Curr Chem 304:323-338

Shin Y, Han S, Jeon JS, Yamamoto K, Zervantonakis IK, Sudo R, Kamm RD, Chung S (2012) Microfluidic assay for simultaneous culture of multiple cell types on surfaces or within hydrogels. Nat Protoc 7(7):1247-1259

Shrestha J, Razavi Bazaz S, Aboulkheyr Es H, Yaghobian Azari D, Thierry B, Ebrahimi Warkiani M, Ghadiri M (2020) Lung-ona-chip: the future of respiratory disease models and pharmacological studies. Crit Rev Biotechnol 40(2):213-230

Sidorov VY, Samson PC, Sidorova TN, Davidson JM, Lim CC, Wikswo JP (2017) I-Wire Heart-on-a-Chip I: three-dimensional cardiac tissue constructs for physiology and pharmacology. Acta Biomater 48:68-78

Snyder LR, Kirkland JJ, Dolan JW (2011) Introduction to modern liquid chromatography. Wiley, Hoboken

Sontheimer-Phelps A, Hassell BA, Ingber DE (2019) Modelling cancer in microfluidic human organs-on-chips. Nat Rev Cancer 19(2):65-81

Srinivasan V, Pamula VK, Pollack MG, Fair RB (2003) Clinical diagnostics on human whole blood, plasma, serum, urine, saliva, sweat, and tears on a digital microfluidic platform. Proc MicroTAS 9:1287-1290

Stein SE (1999) An integrated method for spectrum extraction and compound identification from gas chromatography/mass spectrometry data. J Am Soc Mass Spectrom 10(8):770-781

Sung WC, Makamba H, Chen SH (2005) Chip-based microfluidic devices coupled with electrospray ionization-mass spectrometry. Electrophoresis 26(9):1783-1791

Tabeling P (2005) Introduction to microfluidics. Oxford University Press, Oxford

Tachibana H, Saito M, Tsuji K, Yamanaka K, Tamiya E (2015) Selfpropelled continuous-flow PCR in capillary-driven microfluidic device: microfluidic behavior and DNA amplification. Sens Actuators B Chem 206:303-310

Tan Y-C, Lee AP (2005) Microfluidic separation of satellite droplets as the basis of a monodispersed micron and submicron emulsification system. Lab Chip 5(10):1178-1183

Teh S-Y, Lin R, Hung L-H, Lee AP (2008) Droplet microfluidics. Lab Chip 8(2):198-220

Tenda K, Ota R, Yamada K, Henares TG, Suzuki K, Citterio D (2016) High-resolution microfluidic paper-based analytical devices for sub-microliter sample analysis. Micromachines 7(5):80

Unger MA, Chou H-P, Thorsen T, Scherer A, Quake SR (2000) Monolithic microfabricated valves and pumps by multilayer soft lithography. Science 288(5463):113-116
Upadhyaya S, Selvaganapathy PR (2010) Microfluidic devices for cell based high throughput screening. Lab Chip 10(3):341-348

Van Duinen V, Trietsch SJ, Joore J, Vulto P, Hankemeier T (2015) Microfluidic 3D cell culture: from tools to tissue models. Curr Opin Biotechnol 35:118-126

Vollmer M, Hörth P, Rozing G, Couté Y, Grimm R, Hochstrasser D, Sanchez JC (2006) Multi-dimensional HPLC/MS of the nucleolar proteome using HPLC-chip/MS. J Sep Sci 29(4):499-509

Vrhovski B, Weiss AS (1998) Biochemistry of tropoelastin. Eur J Biochem 258(1):1-18

Wang S, Li E, Gao Y, Wang Y, Guo Z, He J, Zhang J, Gao Z, Wang Q (2013) Study on invadopodia formation for lung carcinoma invasion with a microfluidic 3D culture device. PLoS One 8(2):e56448. https://doi.org/10.1371/journal.pone.0056448

Wang X, Yi L, Mukhitov N, Schrell AM, Dhumpa R, Roper MG (2015) Microfluidics-to-mass spectrometry: a review of coupling methods and applications. J Chromatogr A 1382:98-116

Wang X, Phan DT, Sobrino A, George SC, Hughes CC, Lee AP (2016) Engineering anastomosis between living capillary networks and endothelial cell-lined microfluidic channels. Lab Chip 16(2):282-290

Warkiani ME, Khoo BL, Tan DS-W, Bhagat AAS, Lim W-T, Yap YS, Lee SC, Soo RA, Han J, Lim CT (2014) An ultra-high-throughput spiral microfluidic biochip for the enrichment of circulating tumor cells. Analyst 139(13):3245-3255

Webster A, Greenman J, Haswell SJ (2011) Development of microfluidic devices for biomedical and clinical application. J Chem Technol Biotechnol 86(1):10-17

Weigl BH, Bardell RL, Cabrera CR (2003) Lab-on-a-chip for drug development. Adv Drug Deliv Rev 55(3):349-377

Wheeler AR, Throndset WR, Whelan RJ, Leach AM, Zare RN, Liao YH, Farrell K, Manger ID, Daridon A (2003) Microfluidic device for single-cell analysis. Anal Chem 75(14):3581-3586

Wheeler AR, Moon H, Bird CA, Ogorzalek Loo RR, Kim C-JC, Loo JA, Garrell RL (2005) Digital microfluidics with in-line sample purification for proteomics analyses with MALDI-MS. Anal Chem 77(2):534-540

Wikswo JP, Block FE III, Cliffel DE, Goodwin CR, Marasco CC, Markov DA, McLean DL, McLean JA, McKenzie JR, Reiserer RS (2013) Engineering challenges for instrumenting and controlling integrated organ-on-chip systems. IEEE Trans Biomed Eng 60(3):682-690

Willard HH, Merritt Jr LL, Dean JA, Settle Jr FA (1966) Instrumental methods of analysis. J Chem Educ 43(9):506

Wilmer MJ, Ng CP, Lanz HL, Vulto P, Suter-Dick L, Masereeuw R (2016) Kidney-on-a-chip technology for drug-induced nephrotoxicity screening. Trends Biotechnol 34(2):156-170

Wu Q, Liu J, Wang X, Feng L, Wu J, Zhu X, Wen W, Gong X (2020) Organ-on-a-chip: recent breakthroughs and future prospects. Biomed Eng Online 19(1):9

Wufuer M, Lee G, Hur W, Jeon B, Kim BJ, Choi TH, Lee S (2016) Skin-on-a-chip model simulating inflammation, edema and drugbased treatment. Sci Rep 6:37471. https://doi.org/10.1038/srep3 7471

Xia Y, Si J, Li Z (2016) Fabrication techniques for microfluidic paperbased analytical devices and their applications for biological testing: a review. Biosens Bioelectron 77:774-789

Xie Y, Zhi X, Su H, Wang K, Yan Z, He N, Zhang J, Chen D, Cui D (2015) A novel electrochemical microfluidic chip combined with multiple biomarkers for early diagnosis of gastric cancer. Nanoscale Res Lett 10(1):477

Xu Q, Hashimoto M, Dang TT, Hoare T, Kohane DS, Whitesides GM, Langer R, Anderson DG (2009) Preparation of monodisperse biodegradable polymer microparticles using a microfluidic flow-focusing device for controlled drug delivery. Small 5(13):1575-1581 
Xu Z, Gao Y, Hao Y, Li E, Wang Y, Zhang J, Wang W, Gao Z, Wang Q (2013) Application of a microfluidic chip-based 3D co-culture to test drug sensitivity for individualized treatment of lung cancer. Biomaterials 34(16):4109-4117

Xuan X, Zhu J, Church C (2010) Particle focusing in microfluidic devices. Microfluid Nanofluid 9(1):1-16

Xue Q, Foret F, Dunayevskiy YM, Zavracky PM, McGruer NE, Karger BL (1997) Multichannel microchip electrospray mass spectrometry. Anal Chem 69(3):426-430

Yan S, Zhang J, Chen H, Yuan D, Alici G, Du H, Zhu Y, Li W (2016) Development of a novel magnetophoresis-assisted hydrophoresis microdevice for rapid particle ordering. Biomed Microdevice 18(4):54

Yin H, Marshall D (2012) Microfluidics for single cell analysis. Curr Opin Biotechnol 23(1):110-119

Yin H, Killeen K, Brennen R, Sobek D, Werlich M, van de Goor T (2005) Microfluidic chip for peptide analysis with an integrated HPLC column, sample enrichment column, and nanoelectrospray tip. Anal Chem 77(2):527-533

Yousem SA, Beasley MB (2007) Bronchioloalveolar carcinoma: a review of current concepts and evolving issues. Arch Pathol Lab Med 131(7):1027-1032

Yue S, Xue-Feng Y (2006) Novel multi-depth microfluidic chip for single cell analysis. J Chromatogr A 1117(2):228-233

Zahorodny-Burke M, Nearingburg B, Elias A (2011) Finite element analysis of oxygen transport in microfluidic cell culture devices with varying channel architectures, perfusion rates, and materials. Chem Eng Sci 66(23):6244-6253

Zhang C, Xu J, Ma W, Zheng W (2006) PCR microfluidic devices for DNA amplification. Biotechnol Adv 24(3):243-284

Zhang B, Korolj A, Lai BFL, Radisic M (2018a) Advances in organon-a-chip engineering. Nat Rev Mater 3(8):257-278

Zhang Q, Sito L, Mao M, He J, Zhang YS, Zhao X (2018b) Current advances in skin-on-a-chip models for drug testing. Microphysiol Syst. https://doi.org/10.21037/mps.2018.08.01

Zhao B, Cui X, Ren W, Xu F, Liu M, Ye Z-G (2017) A controllable and integrated pump-enabled microfluidic chip and its application in droplets generating. Sci Rep 7(1):1-8

Zhu P, Tang X, Wang L (2016) Droplet generation in co-flow microfluidic channels with vibration. Microfluid Nanofluid 20(3):47

Ziober BL, Mauk MG, Falls EM, Chen Z, Ziober AF, Bau HH (2008) Lab-on-a-chip for oral cancer screening and diagnosis. Head Neck J Sci Spec Head Neck 30(1):111-121

Publisher's Note Springer Nature remains neutral with regard to jurisdictional claims in published maps and institutional affiliations. 